\title{
Review about mites (Acari) of rubber trees (Hevea spp., Euphorbiaceae) in Brazil $^{1}$
}

\author{
Fábio Akashi Hernandes ${ }^{2}$ \& Reinaldo José Fazzio Feres ${ }^{3}$
}

Biota Neotropica v6 (n1) - http://www.biotaneotropica.org.br/v6n1/pt/abstract?article+bn00406012006

Date Received 06/15/2005

Revised 12/05/2005

Accepted 01/01/2006

\begin{abstract}
${ }^{1}$ Part of Program BIOTA/FAPESP - Instituto Virtual da Biodiversidade, http://www.biota.org.br
${ }^{2}$ Programa de Pós-graduação em Biologia Animal, Bolsista CNPq (fabio akashi@yahoo.com.br).

${ }^{3}$ Departamento de Zoologia e Botânica, Universidade Estadual Paulista. Rua Cristovao Colombo, 2265, Jardim Nazareth, 15054-000 São José do Rio Preto, São Paulo, Brazil (reinaldo@ibilce.unesp.br).
\end{abstract}

\begin{abstract}
Hernandes, F.A. and Feres, R.J.F. Review about mites (Acari) of rubber trees (Hevea spp., Euphorbiaceae) in Brazil. Biota Neotrop . Jan/Abr 2006, vol. 6, no. 1, http://www.biotaneotropica.org.br/v6n1/pt/abstract?article+bn00406012006. ISSN 1676-0611

Two of the most economically important superfamilies of phytophagous mites are Tetranychoidea and Eriophyoidea, which have species represented in rubber trees in Brazil. In this paper we review the literature concerning the mite fauna registered on rubber trees in that country. The source was the information available on literature, but also data from exploratory samplings in Goianésia, State of Goiás, and from a triennial survey with monthly samplings in Cedral, State of São Paulo. Among the phytophagous mites the most important and abundant species were Calacarus heveae and Tenuipalpus heveae. Seven of the nominal species reported belong to the family Tetranychidae. Eutetranychus banksi and Oligonychus gossypii were very numerous in several crops studied, although with no evident damage to the leaves caused by the former. The richest family was Phytoseiidae (27 species). Other rich and numerous family with predatory species was Stigmaeidae (10). The study of mites associated with rubber trees was triggered after the discovery of Calacarus heveae, after what several works arose in order to understand the seasonal occurrence of mites on that culture, their biology, chemical control and influence of associated vegetation. Not surprisingly, most surveys were made in the State of São Paulo, which responds to up to $60 \%$ of the national latex yield. Whereas in some rubber tree crops there were made seasonal samplings, most of the surveys had only few isolated samplings.
\end{abstract}

Key words: Biodiversity, faunistics, mites, rubber tree

\section{Resumo}

Hernandes, F.A. and Feres, R.J.F. Revisão sobre ácaros (Acari) de seringueiras (Hevea spp., Euphorbiaceae) no Brasil. Biota Neotrop. Jan/Abr 2006, vol. 6, no. 1, http://www.biotaneotropica.org.br/v6n1/pt/abstract?article+bn00406012006. ISSN 1676-0611

Duas das superfamílias economicamente mais importantes de ácaros fitófagos são Tetranychoidea e Eriophyoidea, que apresentam espécies presentes no cultivo de seringueira no Brasil. No presente trabalho é revisada a literatura referente à acarofauna registrada em seringueiras no Brasil. O material utilizado foi o disponível na literatura, sendo também incluídos dados de coletas exploratórias esporádicas em Goianésia, Estado de Goiás, e de levantamentos mensais durante três anos em Cedral, noroeste do Estado de São Paulo, Brasil. Entre os ácaros fitófagos, os de maior importância e que ocorreram em maior abundância foram Calacarus heveae e Tenuipalpus heveae. Sete das espécies nominais registradas pertencem à família Tetranychidae. Dentre elas, Eutetranychus banksi e Oligonychus gossypii ocorreram em grande abundância em diversos seringais estudados, a primeira delas, entretanto, sem causar dano aparente às folhas. A família com maior número de espécies (27) foi Phytoseiidae. Outra família com espécies predadoras bastante abundante foi Stigmaeidae (10). O estudo de ácaros associados à seringueira ganhou impulso após a descrição de Calacarus heveae, quando houve incremento dos trabalhos visando melhor compreensão da sazonalidade, biologia, controle químico e influência da vegetação vizinha e associada a seringueiras. A grande maioria das coletas realizadas em seringais paulistas é reflexo do maior número de pesquisadores e da maior produtividade que esse Estado apresenta, respondendo por mais de $60 \%$ da produção nacional de látex. Enquanto que em alguns seringais foram feitas coletas sazonais ao longo do ano, na maioria dos seringais amostrados foram feitas apenas coletas exploratórias pontuais.

Palavras-chave: Ácaros,Biodiversidade, levantamento de fauna,seringueira 


\section{Introduction}

Two of the most economically important superfamilies of phytophagous mites are Tetranychoidea and Eriophyoidea, which have species represented in rubber trees in Brazil. Baker (1945) registered and described the first mite species, Tenuipalpus heveae, from that host. Since then, six other species were described from rubber trees in that country: Calacarus heveae Feres (1992), Phyllocoptruta seringueirae Feres (1998), Shevtchenkella petiolula Feres (1998), Zetzellia agistzellia Hernandes \& Feres (2005), Z. quasagistemas Hernandes \& Feres (2005) and Tetrabdella neotropica Hernandes \& Feres (2006a). Calacarus heveae, considered an important pest of rubber trees, responsible for serious attacks, reaches high populations on the upper side of the leaves from February to April in the northwestern region of the State of São Paulo leading to premature fall of the leaves (Feres 1992, 2000, 2001, Feres et al. 2002, Vieira \& Gomes 1999, Vieira et al. 2000).

The first paper reporting mites of rubber trees (Hevea brasiliensis Muell.Arg., Euphorbiaceae) listed 8 acarine species (Chiavegato 1968). The study of mites associated with that plant was triggered after the discovery of $C$. heveae (Figure 1), after what several works arose in order to understand the diversity and the seasonal occurrence of mites on that crop (Bellini et al. 2005a, Feres 2000, 2001a, 2001b, Feres \& Nunes 2001, Feres et al. 2002, Zacarias \& Moraes 2001, 2002, Ferla \& Moraes 2002a, Hernandes \& Feres 2006b), their biology (Ferla \& Moraes 2003a, Pontier et al. 2000, Hernandes et al. 2006), taxonomy (Feres 1998, Hernandes \& Feres 2005, Pontier \& Flechtmann 1999, 2000), chemical control (Vieira \& Gomes 2001), and influence of associated vegetation (Feres \& Nunes 2001, Bellini et al. 2005b, Demite \& Feres 2005). Tanzini et al.(1999) and Bellini et al.(2005a) registered epizooty of the fungus Hirsutella thompsoni on $C$. heveae. The aim of this work was to summarize the knowledge of mites found on rubber trees in Brazil, giving a panorama of the development of this field and pointing the main species that occur on that plant.

\section{Material and Methods}

In this paper we review the literature concerning the mite fauna registered on rubber trees in Brazil (Hevea spp., Euphorbiaceae), and also include data from exploratory samplings in Goianésia, State of Goiás, (15 $15^{\circ}$ ' 19' S, 48 $57^{\circ} 11^{\prime \prime} \mathrm{W}$ ) and from a triennial survey with monthly samplings conducted from January 2001 to December 2003 in Cedral (Hernandes \& Feres 2006b), northwestern region of the State of São Paulo ( $20^{\circ} 55^{\prime} 30^{\prime \prime}$, $\left.49^{\circ} 26^{\prime} 79^{\prime \prime W}\right)$. Once the material studied by Feres (2000) from Itiquira, State of Mato Grosso, was wrongly reffered in that article as collected in Rondonópolis, State of Mato Grosso (R.J.F. Feres, pers. comm.), the correct location is adopted in the present article.
The nomenclature of the higher taxa follows Woolley (1988), and information concerning the Museum or Institution in which most of the type specimens are deposited is given according to the legends: BMNH - British Museum (Natural History), London, UK; CNC - Canadian National Collection, Toronto, Ontario, Canada; DZSJRP - Coleção de Acari do Departamento de Zoologia e Botânica, UNESP, S.J. do Rio Preto, São Paulo, Brazil; ESALQ - Collection of Departamento de Entomologia, Fitopatologia e Zoologia Agrícola, Universidade de São Paulo/ESALQ, Piracicaba, São Paulo, Brazil; FSCA - Florida State Collection of Arthropods, Gainesville, Florida, USA; IRSN - L’Institut Royal dês Sciences Naturelles, Belgium; LE - Laboratorium voor Entomologie, Landbouwhoogeschool to Wageningen, Netherlands; MCZ - Museum of Comparative Zoology, Harvard University, Cambridge, Massachussetts, USA; NCAPPRI- National Collection of Acari Plant Protection Research Institute, Pretoria, South Africa; UC - University of California, USA; USNM - United States National Museum, Washington DC, USA.

\section{Results and Discussion}

\section{GAMASIDA}

\section{Phytoseiidae Berlese, 1916}

\section{Amblyseius acalyphus Denmark \& Muma, 1973}

Amblyseius acalyphus Denmark \& Muma, 1973: 243; 1989: 75; Moraes et al., 1986: 6; Feres \& Moraes, 1998: 125.

Registers on Hevea: Mato Grosso: Itiquira, Pontes e Lacerda (Ferla \& Moraes 2002a), on H. brasiliensis.

Types: on Acalypha sp (Euphorbiaceae), Rio Claro, São Paulo, Brazil, deposited in ESALQ.

\section{Amblyseius compositus Denmark \& Muma, 1973}

Amblyseius compositus Dermark \& Muma, 1973: 240, 1989:9; Moraes \& McMurtry, 1983: 134.

Registers on Hevea: São Paulo: Piracicaba (Vis et al. 2006), on $H$. brasiliensis.

Types: on Spathodea sp. (Bignoniaceae), Sao Paulo, Brazil, deposited in ESALQ.

\section{Amblyseius impeltatus Denmark \& Muma, 1973}

Amblyseius impeltatus Denmark \& Muma, 1973: 241.

Registers on Hevea: São Paulo: Pariquera-Açu (Zacarias \& Moraes 2001, 2002), on H. brasiliensis.

Types: on Theobroma sp. (Sterculiaceae), Pariquera-Açu, São Paulo, Brazil, deposited in ESALQ. 


\section{Amblyseius neochiapensis Lofego, Moraes \& McMurtry, 2000}

Amblyseius neochiapensis Lofego, Moraes \& McMurtry, 1999 (2000): 462.

Registers on Hevea: Mato Grosso: Itiquira, Pontes e Lacerda (Ferla \& Moraes 2002a), on H. brasiliensis.

Types: on Manihot sp. (Euphorbiaceae), Piritiba, Bahia, Brazil, deposited in ESALQ.

\section{Amblyseius operculatus DeLeon, 1967}

Amblyseius operculatus DeLeon, 1967: 26; Denmark \& Muma, 1989: 47.

Registers on Hevea: São Paulo: Pariquera-Açu (Zacarias \& Moraes 2001, 2002), on H. brasiliensis.

Types: on Cephaelis sp. (Rubiaceae), half way to Simla and Arima, Trinidad, deposited in MCZ.

\section{Amblyseius (Amblyseius) saopaulus Denmark \& Muma, 1973} Amblyseius saopaulus Denmark \& Muma, 1973: 243.

Amblyseius (Amblyseius) saopaulus; Denmark \& Muma, 1989: 32.

Registers on Hevea: São Paulo: Pariquera-Açu (Zacarias \& Moraes 2001, 2002), on H. brasiliensis.

Types: on Theobroma sp. (Sterculiaceae), Pariquera-Açu, São Paulo, Brazil, deposited in ESALQ.

\section{Euseius alatus DeLeon, 1966}

Euseius alatus DeLeon, 1966: 87.

Euseius paraguayensis; Denmark \& Muma, 1970: 224 (synonym according to Moraes \& McMurtry, 1983: 137).

Euseius alatus; Denmark \& Muma, 1973: 262; Moraes \& McMurtry, 1983: 137; Feres \& Moraes, 1998: 127.

Registers on Hevea: São Paulo: Piracicaba (Zacarias \& Moraes 2001, 2002); Mato Grosso: Itiquira, Pontes e Lacerda (Ferla \& Moraes 2002a), on H. brasiliensis.

Types: on Cassia bicapsularis L. (Fabaceae), Georgetown, East Demeraka, Guiana, deposited in collection of D. DeLeon, Erwin, Tennessee, USA.

Remarks: possibly predator of Phyllocoptruta oleivora (Ashmead), a citrus pest (Reis et al. 2000).

\section{Euseius citrifolius Denmark \& Muma, 1970}

Euseius citrifolius Denmark \& Muma, 1970: 222; Moraes \& McMurtry, 1983: 138; Moraes et al., 1991: 131; Feres \& Moraes, 1998: 125; Feres, 2000: 161.

Registers on Hevea: São Paulo: Cedral (Feres et al. 2002, Hernandes \& Feres 2006b), Pindorama, Taquaritinga (Feres et al. 2002), Piracicaba (Zacarias \& Moraes 2001, 2002, Vis et al. 2006), Olímpia (Bellini et al. 2005a), São José do Rio Preto (Demite \& Feres 2005), Ibitinga, Macaubal, Barretos;
Minas Gerais: Frutal (Feres 2000); Mato Grosso: Itiquira (Feres 2000, Ferla \& Moraes 2002a), on H. brasiliensis.

Types: on Citrus sp. (Rutaceae), Asunción, Departamento Central, Paraguai, deposited in FSCA.

Remarks: this is the most frequent and numerous phytoseiid found in rubber trees in the northeastern State of São Paulo (Feres \& Moraes 1998), collected on several host plants.

\section{Euseius concordis (Chant, 1959)}

Typlodromus (Amblyseius) concordis Chant, 1959: 69. Amblyseius (Iphiseius) concordis; Muma, 1961: 288.

Amblyseius concordis; Chant \& Baker, 1965: 22; Moraes \& McMurtry, 1983: 138.

Euseius flechtmanni; Denmark \& Muma, 1970: 223; Denmark \& Muma, 1973: 261 (synonym according to Moraes et al., 1982: 18).

Euseius concordis; Denmark \& Muma, 1973: 264; Moraes \& Oliveira, 1982: 317; Moraes \& McMurtry, 1983: 138; Feres \& Moraes, 1998: 127; Feres 2000: 161.

Registers on Hevea: São Paulo: Cedral (Feres et al. 2002, Hernandes \& Feres 2006b), Taquaritinga (Feres et al. 2002); Mato Grosso: Itiquira (Feres 2000, Ferla \& Moraes 2002a), Pontes e Lacerda (Ferla \& Moraes 2002a), on H. brasiliensis. Types: on Citrus sp. (Rutaceae), Concordia, Entre Rios, Argentina, deposited in USNM.

Remarks: mites of this genus are the most common predatory mites in untreated apple orchards in the State of Rio Grande do Sul, Brazil (Ferla \& Moraes 2002b); the most numerous phytoseiid found in rubber trees in southern State of Mato Grosso (Ferla \& Moraes 2002a).

\section{Galendromimus (Galendromimus) alveolaris (DeLeon, 1957)}

Typhlodromus alveolaris DeLeon, 1957: 141.

Typhlodromus (Typhlodromus) alveolaris; Chant, 1959: 52. Cydnodromella alveolaris; Chant \& Yoshida-Shaul, 1986: 2821; Moraes \& Mesa, 1988: 80.

Galendromimus alveolaris; DeLeon, 1962: 175; DeLeon, 1967: 13; Muma, 1961: 298; Muma et al., 1970: 58.

Galendromimus (Galendromimus) alveolaris; Moraes et al., 1999 (2000): 255.

Registers on Hevea: Mato Grosso: Pontes e Lacerda (Ferla \& Moraes 2002a), on H. brasiliensis.

Types: on Cassia sp. (Fabaceae), Coral Gables, Dade, Florida, USA, deposited in MCZ.

Remarks: abundant in Mabea fistulifera Mart. (Euphorbiaceae) in northeastern State of São Paulo (Daud \& Feres 2005). 


\section{Galendromus (Galendromus) annectens (DeLeon, 1958)}

Typhlodromus annectens DeLeon, 1958: 75; Chant \& Yoshida-Saul, 1984: 1868; Moraes \& McMurtry, 1983: 142; Moraes \& Mesa, 1988: 82; Moraes et al., 1991: 134; Feres \& Moraes, 1998: 128; Feres, 2000: 161; Feres \& Nunes, 2001: 1256.

Galendromus annectens; Muma, 1961: 298; Muma, 1963: 20; Muma et al., 1970: 135; Denmark \& Muma, 1973: 274; Farias et al., 1981: 21; Denmark, 1982: 142; Moraes et al., 1982: 21; Moraes et al., 1986: 186; Gondim Jr. \& Moraes, 2001: 88 .

Galendromus (Galendromus) annectens; Moraes et al., 2004: 265.

Registers on Hevea: Mato Grosso: Itiquira (Feres 2000, Ferla \& Moraes 2002a), Pontes e Lacerda (Ferla \& Moraes 2002a); São Paulo: Olímpia (Bellini et al. 2005a), Cedral (Hernandes \& Feres 2006b), on $H$. brasiliensis.

Types: on Trema floridana Britton ex Small (Ulmaceae), Coral Gables, Dade, Florida, USA, deposited in MCZ.

\section{Galendromus sp.}

Registers on Hevea: Mato Grosso: Pontes e Lacerda (Ferla \& Moraes 2002a), on H. brasiliensis.

\section{Iphiseiodes zuluagai Denmark \& Muma, 1972}

Iphiseiodes zuluagai Denmark \& Muma, 1972: 23.

Amblyseius zuluagai; Moraes \& Mesa, 1988: 79; Moraes et al., 1991: 125.

Iphiseiodes zuluagai; Aponte \& McMurtry, 1995: 165; Kreiter \& Moraes, 1997: 377; Feres \& Moraes, 1998: 127.

Registers on Hevea: São Paulo: Piracicaba (Zacarias \& Moraes 2001, 2002), Taquaritinga (Feres et al. 2002), on $H$. brasiliensis.

Types: on Citrus sinensis (L.) Osbeck (Rutaceae), Palmira, Valle, Colombia, deposited in FSCA.

Remarks: in Taquaritinga, São Paulo, it was collected in a crop neighbor to Citrus sp., where it was also present, probably moving from these cultures (Feres et al. 2002).

\section{Iphiseiodes sp.}

Registers on Hevea: São Paulo: Rio Claro (Flechtmann \& Arleu 1984), on $H$. brasiliensis.

\section{Metaseiulus camelliae (Chant \& Yoshida-Shaul, 1983)}

Typhlodromus camelliae Chant \& Yoshida-Shaul, 1983: 1053; Feres \& Moraes, 1998: 130.

Typhlodromina camelliae; Moraes et al., 1986: 236; Sato et al., 1994: 437; Zacarias \& Moraes, 2001: 583.
Metaseiulus camelliae; Moraes et al. 2004: 278.

Registers on Hevea: São Paulo: Olímpia (Bellini et al2005a), Piracicaba (Zacarias \& Moraes 2002, Vis et al. 2006), Cedral (Hernandes \& Feres 2006b), on H. brasiliensis.

Types: on Camellia sp. (Theaceae), Uruguai, intercepted in Miami, Florida, USA, deposited in CNC.

\section{Neoseiulus anonymus (Chant \& Baker, 1965)}

Amblyseius anonymus Chant \& Baker, 1965: 21; Schicha \& Elshafie, 1980: 32; McMurtry, 1983: 254.

Neoseiulus anonymus; Denmark \& Muma, 1973: 27; Moraes \& Mesa, 1988: 76; Moraes et al., 1991: 126; Kreiter \& Moraes, 1997: 378; Moraes et al., 1999 (2000): 245.

Registers on Hevea: Mato Grosso: Itiquira, Pontes e Lacerda (Ferla \& Moraes 2002a), on H. brasiliensis.

Types: on banana (Musa paradisiaca L., Musaceae), Tacamiche, La Lima, Honduras, deposited in USNM.

Remarks: one of the species commonly associated with Mononychellus tanajoa (Bondar) (Tetranychidae) in Northeastern Brazil (Moraes et al.1988).

\section{Neoseiulus idaeus Denmark \& Muma, 1973}

Neoseiulus idaeus Denmark \& Muma, 1973: 266.

Amblyseius idaeus; Moraes \& McMurtry, 1983: 134.

Registers on Hevea: São Paulo: Cedral (Hernandes \& Feres 2006b), on H. brasiliensis.

Types: on Rubus idaeus L. (Rosaceae), Piracicaba, São Paulo, Brazil, deposited in ESALQ.

\section{Neoseiulus tunus (DeLeon, 1967)}

Typhlodromips tunus DeLeon, 1967: 29; Denmark \& Muma, 1973: 253; Moraes et al., 1986: 151.

Amblyseius tunus; McMurtry \& Moraes, 1989: 181; Feres \& Moraes, 1998: 126.

Neoseiulus tunus; Ferla \& Moraes, 2002a: 872; Moraes et al., 2004: 148.

Registers on Hevea: Mato Grosso: Pontes e Lacerda (Ferla \& Moraes 2002a), on H. brasiliensis.

Types: on guava (Psidium guajava L., Mirtaceae), Upper Saint John’s Road, Tunapuna, Trinidad, deposited in MCZ.

\section{Phytoscutus sexpilis Muma, 1961}

Phytoscutus sexpilis Muma, 1961: 275; DeLeon, 1967: 17.

Typhlodromus sexpilis; Hirschmann, 1962: 17.

Amblyseius sexpilis; van der Merwe, 1968: 161.

Phytoscutus sexpilis; Muma et al., 1970: 24; Yoshida-Shaul \& Chant, 1997: 234. 
Registers on Hevea: São Paulo: Olímpia (Bellini et al. 2005a), Pariquera-Açu (Zacarias \& Moraes 2001, 2002), on $H$. brasiliensis.

Types: on grapefruit (Citrus paradisi MacFad., Rutaceae), Polk City, Florida, USA, deposited in USNM.

\section{Proprioseiopsis dominigos (El-Benhawy, 1984)}

Amblyseius dominigos El-Benhawy, 1984: 130; McMurtry \& Moraes, 1989: 185; Moraes et al., 1991: 126; Feres \& Moraes, 1998: 126.

Proprioseiopsis dominigos; Gondim Jr. \& Moraes, 2001: 81. Registers on Hevea: São Paulo: Pariquera-Açu (Zacarias \& Moraes 2001, 2002), on H. brasiliensis.

Types: on unidentified plant, Sooretama, Espírito Santo, Brazil, deposited in CNC.

\section{Proprioseiopsis ovatus (Garman, 1958)}

Amblyseiopsis ovatus Garman, 1958: 78.

Amblyseius ovatus, Moraes \& McMurtry, 1983: 133; Moraes et al., 1991: 127.

Typhlodromus (Amblyseius) ovatus, Chant, 1959: 90.

Proprioseiopsis ovatus, Denmark \& Muma, 1973: 237.

Registers on Hevea: São Paulo: Piracicaba (Vis et al. 2006), on $H$. brasiliensis.

Types: on Cattleya sp. (Orchidaceae), from Equador at Brownsville, Texaz, deposited in USNM.

Typhlodromalus feresi Lofego, Moraes \& McMurtry, 2000 Typhlodromalus feresi Lofego, Moraes \& McMurtry, (1999) 2000: 466.

Registers on Hevea: Mato Grosso: Pontes e Lacerda (Ferla \& Moraes 2002a), on H. brasiliensis.

Types: on Mabea sp. (Euphorbiaceae) (M. fistulifera Mart., R.J.F. Feres, com. pess.), São José do Rio Preto, São Paulo, Brazil, deposited in ESALQ.

\section{Typhlodromalus aff. horatii}

Registers on Hevea: Mato Grosso: Pontes e Lacerda (Ferla \& Moraes 2002a), on H. brasiliensis.

\section{Typhlodromips amilus DeLeon, 1967}

Typhlodromips amilus DeLeon, 1967: 28.

Registers on Hevea: Mato Grosso: Pontes e Lacerda (Ferla \& Moraes 2002a), on H. brasiliensis.

Types: on Bromeliaceae, Simla, Trinidad, deposited in MCZ.

Typhlodromips cananeiensis Gondim Jr. \& Moraes, 2001
Typhlodromips cananeiensis Gondim Jr. \& Moraes, 2001: 84.

Registers on Hevea: São Paulo: Cananéia (Zacarias \& Moraes 2001), Pariquera-Açu (Zacarias \& Moraes 2002), on H. brasiliensis.

Types: on Bactris setosa Mart. (Arecaceae), Cananéia, São Paulo, Brazil, deposited in ESALQ.

\section{Typhlodromips aff. sinensis}

Registers on Hevea: Mato Grosso: Pontes e Lacerda (Ferla \& Moraes 2002a), on H. brasiliensis.

Typhlodromus (Anthoseius) transvaalensis (Nesbitt, 1951) Kampimodromus transvaalensis Nesbitt, 1951: 55.

Typhlodromus transvaalensis; Chant, 1955: 498.

Typhlodromus jackmickleyi; DeLeon, 1958: 175.

Typhlodromus pectinatus; Athias-Henriot, 1958: 179.

Neoseiulus transvaalensis; Muma, 1961: 295.

Clavidromus jackmickleyi; Muma, 1961: 296.

Clavidromus transvaalensis; Muma \& Denmark, 1968: 238.

Typhlodromus (Anthoseius) transvaalensis; Chant \& McMurtry, 1994: 252.

Registers on Hevea: Mato Grosso: Pontes e Lacerda (Ferla \& Moraes 2002a), on H. brasiliensis.

Types: on peanut (Arachis hypogaea L., Fabaceae), Nylstroom, Transvaal, South Africa, deposited in CNC.

\section{ACTINEDIDA}

\section{Acarophenacidae Cross, 1965}

Unidenfified sp.

Registers on Hevea: São Paulo: São Jose do Rio Preto (Demite \& Feres 2005) on $H$. brasiliensis. Remarks: parasites on insects; casual record on rubber trees.

\section{Bdellidae Dugès, 1834}

\section{Tetrabdella neotropica Hernandes \& Feres, 2006a:57}

Tetrabdella neotropica Hernandes \& Feres, 2006a:57

Registers on Hevea: São Paulo: Cedral (Feres et al. 2002, Hernandes \& Feres 2006b), Pindorama (Feres et al. 2002), Olímpia (Bellini et al. 2005a), Piracicaba (Vis et al. 2006), São Jose do Rio Preto (Demite \& Feres 2005), on H. brasiliensis. Remarks: Previously referred as Spinibdella sp. (Feres et al. 2002, Bellini et al. 2005a), and aff. Spinibdella (Demite \& Feres 2005, Vis et al. 2006), this genus bears only two pairs of trichobothriae, on tarsi III and IV (Hernandes \& Feres 2005b). As in Spinibdella cronini Baker \& Balock it spins a silken cocoon around each egg (Wallace \& Mahon 1972); in rubber trees it occurs mostly at the base of the leaflets, where it spins a silken cocoon, inside which it moults. 


\section{Cheyletidae Leach, 1815}

Cheletomimus (Hemicheyletia) wellsi (Baker, 1949a)

Cheyletia wellsi Baker, 1949a: 300-301.

Paracheyletia wellsi; Volgin, 1955: 152; Muma, 1964: 245-246.

Dendrocheyla wellsi; Volgin, 1969: 211.

Hemicheyletia wellsi; Summers \& Price, 1970: 18.

Cheletomimus (Hemicheyletia) wellsi; Fain et al. 2002: 45; Feres 2000: 162.

Registers on Hevea: São Paulo: Reginópolis (Feres 2000), Taquaritinga (Feres et al. 2002), Cedral (Hernandes \& Feres 2006b), on $H$. brasiliensis.

Types: on Citrus sp. (Rutaceae), Philadelfia, USA, deposited in USNM.

Remarks: this predator is commonly found in leaves and fruits of Citrus attacked by Phyllocoptruta oleivora (Ashmead) (Chiavegato 1980).

\section{Cheletogenes sp.}

Registers on Hevea: São Paulo: Olímpia (Bellini et al. 2005a); Goiás: Goianésia, on H. brasiliensis.

\section{Cheyletia sp.}

Registers on Hevea: São Paulo: Olímpia (Bellini et al. 2005a); Goiás: Goianésia, on H. brasiliensis.

\section{Hemicheyletia sp.}

Registers on Hevea: Pariquera-Açu (Zacarias \& Moraes 2002), on H. brasiliensis.

\section{Cunaxidae Thor, 1902}

\section{Pulaeus sp.}

Registers on Hevea: São Paulo: Pariquera-Açu (Zacarias \& Moraes 2002), on H. brasiliensis.

\section{Pseudobonzia sp.}

Registers on Hevea: Mato Grosso: Itiquira (Feres 2000, Ferla \& Moraes 2002a), Pontes e Lacerda (Ferla \& Moraes 2002a); São Paulo: Cedral, on H. brasiliensis.

\section{Scutopalus sp.}

Pariquera-Açu (Zacarias \& Moraes 2002), on H. brasiliensis.

\section{Eriophyidae Nalepa, 1898}

\section{aff. Acaphyllisa sp.}

Registers on Hevea: Mato Grosso: Itiquira (Ferla \& Moraes 2002a), on H. brasiliensis.

Remarks: probably casual record on rubber trees; only one

\section{Calacarus heveae Feres, 1992}

Calacarus heveae Feres, 1992: 61; 2000: 167; 2001: 343.

Registers on Hevea: Amazonas: Manaus (Feres 2001a); São Paulo: Cedral (Feres et al. 2002, Hernandes \& Feres 2006b), Pindorama, Taquaritinga (Feres et al. 2002), Olímpia (Bellini et al. 2005a), Pariquera-Açu (Zacarias \& Moraes 2002), Piracicaba (Zacarias \& Moraes 2002, Vis et al. 2006), São José do Rio Preto (Demite \& Feres 2005), Américo de Campos, Barretos, José Bonifácio, Pindorama, Planalto, Macaubal, Monte Aprazível, Reginópolis; Minas Gerais: Frutal (Feres 2000); Mato Grosso: Itiquira (Feres 2000, Ferla \& Moraes 2002a), Pontes e Lacerda (Ferla \& Moraes 2002a); Mato Grosso do Sul: Selvíria (Feres 2000), Goiás: Goianésia, on $H$. brasiliensis.

Types: on H. brasiliensis (Euphorbiaceae), Planalto, São Paulo, Brazil, deposited in DZSJRP.

Remarks: serious pest of rubber trees in Brazil, this species reaches large populations on the upper side of the leaves from January to April; it leads to premature fall of leaves, preceding the natural senescence, bringing on extra budding (Feres 1992, 2000, 2001, Vieira \& Gomes 1999).

\section{aff. Chakrabartiella sp.}

Registers on Hevea: Mato Grosso: Pontes e Lacerda (Ferla \& Moraes 2002a), on H. brasiliensis.

Remarks: probably casual record on rubber trees, only four individuals were collected.

\section{Phyllocoptruta seringueirae Feres, 1998}

Phyllocoptruta seringueirae Feres, 1998: 71; 2000: 168; 2001: 343.

Registers on Hevea: Amazonas: Manaus (Feres 2001a); São Paulo: Cedral (Feres et al. 2002, Hernandes \& Feres 2006b), Pindorama (Feres et al. 2002), Olímpia (Bellini et al. 2005a), Buritama, Monte Aprazível, Reginópolis (Feres 2000), São José do Rio Preto (Demite \& Feres 2005); Mato Grosso: Itiquira (Feres 2000, Ferla \& Moraes 2002a), Pontes e Lacerda (Ferla \& Moraes 2002a); Goiás: Goianésia, on H. brasiliensis. Types: on H. brasiliensis Muell. Arg. (Euphorbiaceae), Reginópolis, São Paulo, Brazil, deposited in DZSJRP.

Remarks: registered in great abundance in rubber tree crops of Itiquira, Mato Grosso (Ferla \& Moraes 2002a, R.J.F. Feres, pers. comm.).

\section{Shevtchenkella petiolula Feres, 1998}

Shevtchenkella petiolula Feres, 1998: 69; 2000: 168; 2001: 343.

Registers on Hevea: Amazonas: Manaus (Feres 2001a); São Paulo: Cedral (Feres et al. 2002, Hernandes \& Feres 2006b), Pindorama (Feres et al. 2002), Olímpia (Bellini et al. 2005a), Piracicaba (Vis et al. 2006), Buritama, Reginópolis (Feres 
2000), São José do Rio Preto (Demite \& Feres 2005); Mato Grosso: Itiquira (Feres 2000, Ferla \& Moraes 2002a), Pontes e Lacerda (Ferla \& Moraes 2002a); Mato Grosso do Sul: Selvíria (Feres 2000), on H. brasiliensis.

Types: on H. brasiliensis Muell. Arg. (Euphorbiaceae), Buritama, São Paulo, Brazil, deposited in DZSJRP.

Remarks: this species is mostly found on petioles, petiolules and flowers of rubber trees, in small number, from September to November (Feres 2000).

\section{Eupalopsellidae Willmann, 1952}

Exothorhis caudata Summers, 1960

Exothorhis caudata Summers, 1960: 131; Rakha \& McCoy, 1985: 142.

Exothorhis citri, Meyer \& Ueckermann, 1989: 10.

Exothorhis caudata, Rimando \& Corpuz-Raros, 1996: 110; Swift, 1997: 39.

Registers on Hevea: São Paulo: Piracicaba (Vis et al. 2006), on $H$. brasiliensis.

Types: on Citrus sp. (Rutaceae), Florida, USA, deposited in UC, USA.

Remarks: Matioli et al.(2002) suggested this species reproduce by parthenogenesis, because no males have been registered to date. It was found associated with some citrus scale insects (Parlatoria cinerea, Coccus viridis, Saissetia coffeae, Selenaspidus articulatus, Orthezia praelonga and Pinnaspis aspidistrae)

\section{Stigmaeidae Oudemans, 1931}

Agistemus floridanus Gonzalez-Rodriguez, 1965

Agistemus floridanus Gonzalez-Rodriguez, 1965: 38; Matioli et al., 2002: 103; Arruda Filho \& Moraes, 2003: 52.

Registers on Hevea: Mato Grosso: Itiquira, Pontes e Lacerda (Ferla \& Moraes 2002a); São Paulo: Olímpia (Bellini et al. 2005a) on H. brasiliensis.

Types: on Ligustrum sp. (Oleaceae), Orlando, Florida, USA, deposited in USNM.

Remarks: this species showed high oviposition rate when fed on $C$. heveae and T. heveae, serious pests of rubber trees in Brazil (Ferla \& Moraes 2003a); along with Phytoseiidae, mites of this family are the most commonly found predators in rubber trees of Southeast and Middle West Brazil (Feres 2000, Ferla \& Moraes 2002a).

\section{Agistemus aff. floridanus}

Registers on Hevea: Cedral (Hernandes \& Feres 2006b).

Remarks: as mentioned by Buosi et al. (2006), there is a large range in several body measurements of this species, comprising the values reported by Matioli et al. (2002) for
A. brasiliensis Matioli et al. (2002) and A. floridanus Gonzalez (1965). In that respect, it was not possible to determinate the real status of that species.

\section{Agistemus sp.}

Registers on Hevea: São Paulo: Ibitinga, Barretos (Feres 2000), Cedral, Pindorama (Feres et al. 2002), Pariquera-Açu (Zacarias \& Moraes 2002), Piracicaba (Zacarias \& Moraes 2002, Vis et al. 2006); Mato Grosso: Itiquira (Feres 2000); Goiás: Goianésia, on H. brasiliensis; Barretos (Feres 2000) on $H$. pauciflora e $H$. benthamiana.

Remarks: species of this genus are often cited as predators of tetranychid eggs (McMurtry et al.1970, Oomen 1982, Inoue \& Tanaka 1983 apud Ehara 1993) and of species of Tenuipalpus (Flechtmann 1975); occurs in a large variety of plants. Probably some registers of Agistemus males are from males of Zetzellia agistzellia.

\section{Eryngiopus sp.}

Registers on Hevea: São Paulo: Piracicaba (Vis et al. 2006), on $H$. brasiliensis.

\section{Eustigmaeus sp.}

Registers on Hevea: São Paulo: Piracicaba (Zacarias \& Moraes 2002), on H. brasiliensis.

Remarks: referred as Ledermuelleria sp., synonym by Wood (1973:182).

\section{Mediolata sp.}

Registers on Hevea: São Paulo: Piracicaba (Vis et al. 2006), on $H$. brasiliensis.

Zetzellia malvinae Matioli, Ueckermann \& Oliveira, 2002 Zetzellia malvinae Matioli, Ueckermann \& Oliveira, 2002: 111.

Registers on Hevea: São Paulo: Piracicaba (Vis et al. 2006), on $H$. brasiliensis.

Types: on Citrus sinensis (Rutaceae), Limeira, Sao Paulo, Brazil, deposited in ESALQ.

\section{Zetzellia mapuchina Gonzalez-Rodriguez, 1965}

Zetzellia mapuchina Gonzalez-Rodriguez, 1965: 23.

Registers on Hevea: São Paulo: Cedral (Hernandes \& Feres 2006b), on $H$. brasiliensis.

Types: on Citrus reticulata (Rutaceae), Argentina, intercepted in Miami, Florida, USA, deposited in USNM.

\section{Zetzellia agistzellia Hernandes \& Feres, 2005}


Zetzellia agistzellia Hernandes \& Feres, 2005: 28.

Registers on Hevea: São Paulo: Cedral (Hernandes \& Feres 2006b), on H. brasiliensis.

Remarks: This species present sexes with different patterns of organization of dorsal platelets: males resemble Agistemus whereas females resemble Zetzellia.

\section{Zetzellia quasagistemas Hernandes \& Feres, 2005}

Zetzellia quasagistemas Hernandes \& Feres, 2005: 37.

Registers on Hevea: Mato Grosso: Itiquira, Pontes e Lacerda (Ferla \& Moraes 2002a); São Paulo: Ibitinga, Reginópolis (Feres 2000), Olímpia (Bellini et al. 2005a), Pindorama (Feres et al. 2002), São José do Rio Preto (Demite \& Feres 2005), Cedral (Feres et al. 2002, Hernandes \& Feres 2006b); Goiás: Goianésia, on $H$. brasiliensis.

Remarks: Previously referred as Zetzellia sp. (Bellini et al. 2005a) and Z. aff. yusti (Ferla \& Moraes 2002a), this species present, as Z. agistzellia, some characters commonly found in males of Agitemus: males with setae $f 1$ inserted on the main plate and setae $e 1$ greatly reduced.

\section{Tarsonemidae Canestrini \& Fanzago, 1877}

Daidalotarsonemus spp.

Registers on Hevea: Mato Grosso: Itiquira (Feres 2000); São Paulo: Piracicaba (Vis et al. 2006), on H. brasiliensis.

\section{Fungitarsonemus sp.}

Registers on Hevea: São Paulo: Pindorama (Feres et al. 2002), Olímpia (Bellini et al. 2005a), Cedral (Hernandes \& Feres 2006b), on $H$. brasiliensis.

\section{Polyphagotarsonemus latus (Banks, 1904)}

Tarsonemus latus Banks, 1904: 1553.

Hemitarsonemus latus; Ewing, 1939: 54.

Neotarsonemus latus; Smiley, 1967: 137.

Polyphagotarsonemus latus; Beer \& Nucifora, 1965: 38; Feres 2000: 164.

Registers on Hevea: Minas Gerais: Frutal (Feres 2000); São Paulo: Pindorama (Feres et al. 2002), Campinas (Chiavegato 1968); Mato Grosso: Itiquira, Pontes e Lacerda (Ferla \& Moraes 2002a), on H. brasiliensis.

Types: on mango (Mangifera sp., Anacardiaceae), Washington DC, USA., in greenhouse, deposited at MCZ.

Remarks: cosmopolitan pest of several crops; in Campinas, it was collected in clones of rubber trees (Chiavegato 1968).

\section{Tarsonemus confusus Ewing, 1939}

Tarsonemus confusus Ewing, 1939: 26; Smiley, 1969: 221; Kaliszewski, 1993: 40.

Registers on Hevea: São Paulo: Olímpia (Bellini et al. 2005a), on $H$. brasiliensis.

Types: on Delphinium belladonna, (Ranunculaceae), Suitland, MD, USA, deposited in USNM.

Remarks: this species has been found in both thelytokous and sexual populations (Lindquist 1986 apud Wrensch \& Ebbert 1993).

\section{Tarsonemus spp.}

Registers on Hevea: Mato Grosso: Itiquira (Feres 2000, Ferla \& Moraes 2002a), Pontes e Lacerda (Ferla \& Moraes 2002a); São Paulo: Cedral (Feres et al. 2002, Hernandes \& Feres 2006b), Pindorama (Feres et al. 2002), Piracicaba (Vis et al. 2006), São José do Rio Preto (Demite \& Feres 2005), Pariquera-Açu (Zacarias \& Moraes 2002), on H. brasiliensis.

Remarks: it may be more than one species, for the material from Pontes e Lacerda was not examined; mites of this genus are usually cosmopolitans and primarily mycophagous.

\section{Xenotarsonemus sp.}

Registers on Hevea: São Paulo: Pariquera-Açu (Zacarias \& Moraes 2002), on Hevea brasiliensis.

\section{Tenuipalpidae Berlese, 1913}

Brevipalpus phoenicis (Geijskes, 1939)

Tenuipalpus phoenicis Geijskes, 1939: 23.

Brevipalpus phoenicis; Sayed, 1946a: 99.

Brevipalpus yothersi; Baker, 1949b: 374.

Brevipalpus mcbridei; Baker, 1949b: 374.

Brevipalpus papayensis; Baker, 1949b: 379.

Brevipalpus phoenicis; Pritchard \& Baker, 1958: 233; DeLeon, 1961: 48; Gonzalez, 1975: 82; Baker et al., 1975: 18; Meyer, 1979: 87; Baker \& Tuttle, 1987: 9899; Feres 2000: 164.

Registers on Hevea: São Paulo: Cedral (Feres 2000, Hernandes \& Feres 2006b), Ibitinga, José Bonifácio, Macaubal, Reginópolis (Feres 2000), Pindorama (Feres et al. 2002), Piracicaba (Vis et al. 2006), Rio Claro; Bahia: Itabuna (Flechtmann \& Abreu 1973; Flechtmann \& Arleu 1984); Mato Grosso: Itiquira, Pontes e Lacerda (Ferla \& Moraes 2002a), on H. brasiliensis.

Types: on Phoenix sp. (Arecaceae), Netherlands, in greenhouse, deposited in LE.

Remarks: one of the most serious pests of citrus, bearer of leprosis virus; reponsible for leaf fall and low quality of coffee (Chagas 1973): cosmonolitan. collected from un to 
100 host plants only in Central America (Childers et al. 2001).

\section{Brevipalpus sp.}

Registers on Hevea: São Paulo: Pariquera-Açu (Zacarias \& Moraes 2001), on H. brasiliensis.

\section{Tenuipalpus heveae Baker, 1945}

Tenuipalpus heveae Baker, 1945: 36; Baker \& Pritchard, 1953 : 320; Feres, 2000: 165.

Registers on Hevea: São Paulo: Cedral (Feres et al. 2002, Hernandes \& Feres 2006b), Pindorama, Taquaritinga (Feres et al. 2002); Barretos, Pindorama (Feres 2000), Cedral, Olímpia (Bellini et al. 2005a), Piracicaba (Vis et al. 2006), São José do Rio Preto (Demite \& Feres 2005); Mato Grosso: Itiquira (Feres 2000, Ferla \& Moraes 2002a), Pontes e Lacerda (Ferla \& Moraes 2002a); Amazonas: Manaus (Flechtmann \& Arleu 1984); Pará: Belém (Flechtmann 1979); Goiás: Goianésia, on H. brasiliensis; Itiquira, on $H$. viridis (Feres 2000), on $H$. viridis.

Types: on H. brasiliensis (Euphorbiaceae), Belterra, Pará, Brazil, deposited in USNM.

Remarks: registered in large populations mostly on the lower side of the leaves (Feres 2000, Feres et al. 2002, Ferla \& Moraes 2002a); but also found on the upper side when in high infestation; in December 2000, it led to bronzing and severe fall of the leaves in crops from Goianésia, Goiás (J.F.C. Benesi, pers. comm.).

\section{Tetranychidae Donnadieu, 1875}

\section{Allonychus braziliensis (McGregor, 1950)}

Septanychus braziliensis McGregor, 1950: 318.

Allonychus braziliensis; Pritchard \& Baker, 1955: 137.

Registers on Hevea: São Paulo: Campinas (Chiavegato 1968), on $H$. brasiliensis.

Types: on quince (Cydonia sp., Rosaceae), Viçosa, Minas Gerais, Brazil, deposited in USNM.

Remarks: possibly casual record, on clones of rubber trees (Chiavegato 1968).

\section{Atrichoproctus uncinatus Flechtmann, 1967}

Atrichoproctus uncinatus Flechtmann, 1967: 39 apud Flechtmann \& Baker, 1970: 157; Flechtmann \& Baker, 1975: 116; Feres, 2000: 166.

Registers on Hevea: Mato Grosso: Itiquira, on $H$. benthamiana (Feres 2000).

Types: on Rhododendron indicum (L.) Sweet, (Ericaceae), Desmodium sp. (Fabaceae) and Quercus sp. (Fagaceae), Piracicaba and Matão, São Paulo, Brazil, deposited in ESALQ. Remarks.greentcolored species, occurs mostly on the up- per side of the leaves.

\section{Eutetranychus banksi (McGregor, 1914)}

Tetranychus banksi McGregor, 1914: 358.

Neotetranycus banksi; (McGregor) Banks, 1917: 177.

Anychus banksi; (McGregor) McGregor, 1919: 644.

Eutetranychus banksi; (McGregor) McGregor, 1950: 141.

Tetranychus rusti; McGregor, 1917: 582.

Anychus rusti; (McGregor) McGregor, 1919: 645.

Eutetranychus rusti; (McGregor) McGregor, 1950: 669; synonym according to Pritchard \& Baker (1955).

Anychus clarki; McGregor, 1935: 161.

Eutetranychus clarki; (McGregor) McGregor, 1950: 270; synonym according to Pritchard \& Baker (1955).

Anychus orientalis; Klein, 1936: 3.

Anychus ? latus; Hirst, 1923: 991.

Anychus latus; Sayed, 1946c: 125.

Anychus africanus; Tucker, 1926: 5.

Anychus verganii; Blanchard, 1940: 24; synonym according to Pritchard \& Baker (1955).

Anychus ricini; Rahman \& Sapra, 1940: 194.

Eutetranychus mexicanus; McGregor, 1950: 27; synonym according to Pritchard \& Baker (1955).

Eutetranychus banksi; McGregor, 1914: 268; Pritchard \& Baker, 1955: 115; Flechtmann \& Baker, 1970: 156; Flechtmann \& Baker, 1975: 112; Feres 2000: 165.

Registers on Hevea: São Paulo: Campinas (Chiavegato 1968), Cedral (Feres et al. 2002, Hernandes \& Feres 2006b), Pindorama, Taquaritinga (Feres et al. 2002), Olímpia (Bellini et al. 2005a), Pariquera-Açu (Zacarias \& Moraes 2002), Piracicaba (Zacarias \& Moraes 2002, Vis et al. 2006), São José do Rio Preto (Demite \& Feres 2005), Bálsamo, Ibitinga, Macaubal, Pindorama; Minas Gerais: Frutal (Feres 2000); Mato Grosso: Itiquira (Ferla \& Moraes 2002a), on $H$. brasiliensis.

Types: on castor bean (Ricinus communis L., Euphorbiaceae) and Stizolobium sp. (Fabaceae), Orlando, Florida, USA, deposited in USNM.

Remarks: collected from many hosts all around the world (Bolland et al.1998); pest of citrus in USA.; registered in great abundance in rubber trees in Brazil, although with no evident damage to the leaves; in Campinas, it was collected in yards of clones of rubber trees (Chiavegato 1968).

\section{Mixonychus sp.}

Registers on Hevea: São Paulo: Piracicaba (Vis et al. 2006), on $H$. brasiliensis.

\section{Mononychellus sp.}

Registers on Hevea: Mato Grosso: Itiquira, Pontes e Lacerda 
(Ferla \& Moraes 2002a), on H. brasiliensis.

\section{Oligonychus coffeae (Nietner, 1861)}

Acarus coffeae Nietner, 1861: 19.

Tetranychus bioculatus; Wood-Mason, 1884: 1.

Paratetranychus bioculatus; Baker \& Pritchard, 1953: 213.

Oligonychus merwei; Tucker, 1926: 6.

Paratetranychus terminalis; Sayed, 1946b: 94.

Oligonychus coffeae; Pritchard \& Baker, 1955: 315; Baker \& Pritchard, 1960: 505; Meyer \& Rodrigues, 1965: 12; Rodrigues, 1968: 220; Gutierrez, 1968: 446; Meyer, 1974: 251; Meyer, 1987: 146; Feres 2000: 166;

Registers on Hevea: Mato Grosso: Itiquira, Pontes e Lacerda (Ferla \& Moraes 2002a), on $H$. brasiliensis; Itiquira, on $H$. guianensis and $H$. viridis (Feres 2000).

Types: on Coffea arabica L. (Rubiaceae), Sri Lanka (Ceylon), institution of deposition of types not found.

Remarks: considered the most serious pest of tea in several countries (Flechtmann \& Arleu 1984); on rubber trees, however, it has not been observed great damages.

\section{Oligonychus gossypii (Zacher, 1921)}

Paratetranychus gossypii Zacher, 1921: 183; Hirst, 1926: 832; André, 1933: 306.

Oligonychus gossypii; Pritchard \& Baker, 1955: 359; Baker \& Pritchard, 1960: 508; Baker \& Pritchard, 1962(1963): 327; Flechtmann, 1967: 23, 31; Meyer, 1974: 263; Meyer, 1987: 152; Feres, 2000: 166.

Registers on Hevea: São Paulo: Cedral (Feres et al. 2002, (Hernandes \& Feres 2006b), Ibitinga, Barretos (Feres 2000), Piracicaba (Zacarias \& Moraes 2002, Vis et al. 2006); Mato Grosso: Itiquira (Feres 2000, Ferla \& Moraes 2002a), Pontes e Lacerda (Ferla \& Moraes 2002a); Espírito Santo: Viana (Flechtmann \& Arleu 1984); Acre: Rio Branco (Flechtmann 1989; Fazolin \& Pereira 1989); Amazonas: Manaus (Fazolin \& Pereira 1989), on H. brasiliensis; Itiquria, Mato Grosso (Feres 2000), on $H$. rigidifolia and $H$. viridis.

Types: on cotton (Gossypium herbaceum L., Malvaceae), Togo, Africa, probably deposited in Zacher's collection..

Remarks: registered in great abundance in crops of the States of Pará and Amazonas, leading to intense bronzing and premature fall of the leaves (Fazolin \& Pereira 1989, Flechtmann 1989); reported on many plants in West Africa and Central America, as cotton, cassava, bean and papaya (Pritchard \& Baker 1955).

\section{Tetranychus mexicanus (McGregor, 1950)}

Septanychus mexicanus McGregor, 1950: 323.

Tetranychus mexicanus; Pritchard \& Baker, 1955: 411;
Flechtmann, 1967: 21, 29; Chiavegato, 1968: 67; Flechtmann \& Baker, 1970: 162; Flechtmann \& Baker, 1975: 120; Feres, 2000: 167.

Registers on Hevea: Mato Grosso: Itiquira (Feres 2000, Ferla \& Moraes 2002a), Pontes e Lacerda (Ferla \& Moraes 2002a); São Paulo: Campinas (Chiavegato 1968), on H. brasiliensis; Itiquira, on H. benthamiana and H. pauciflora (Feres 2000).

Types: on Citrus (Rutaceae), Mexico, intercepted in Laredo, Texas, USA, deposited in USNM.

Remarks: species of broad geographical distribution, collected from many host plants; in rubber trees it occurs mostly on the lower side of the leaves, where it produces considerable amounts of silk; in Campinas, it was collected in yards of clones of rubber trees (Chiavegato 1968).

\section{Tetranychus urticae Koch, 1836}

Tetranychus urticae Koch, 1836: 10.

Acarus telarius Linnaeus; 1758: 616.

Tetranychus telarius (L.); Dugès, 1834: 15; synonymy according to Smith \& Baker (1968).

Acarus sambuci Schrank, 1781: 521.

Tetranychus sambuci (Schrank); Koch, 1842: 37.

Epitetranychus sambuci (Schrank); Oudemans, 1931a: 194; synonymy according to Pritchard \& Baker (1955).

Tetranychus dugesii; Cano \& Alcacio, 1886: 197; synonymy according to Estebanes \& Baker (1968).

Acarus textor; Fourcroy, 1785: 530.

Tetranychus textor (Fourcroy); Oudemans, 1929: 276; synonymy according to Pritchard \& Baker (1955).

Tetranychus russeolus; Koch, 1838: 15; synonymy according to Pritchard \& Baker (1955).

Tetranychus viburni; Koch, 1838: 17.

Schizotetranychus viburni; (Koch) Oudemans, 1937: 1061; synonymy according to Pritchard \& Baker (1955).

Tetranychus fervidus; Koch, 1842: 21; synonymy according to Pritchard \& Baker (1955).

Acarus cucumeris; Boisduval, 1867: 84.

Tetranychus cucumeris; (Boisduval) Murray, 1877: 102; synonymy according to Pritchard \& Baker (1955).

Acarus rosarum; Boisduval, 1867: 84.

Tetranychus rosarum; (Boisduval) Murray, 1877: 102; synonymy according to Pritchard \& Baker (1955).

Acarus cinnabarinus; Boisduval, 1867: 88

Tetranychus cinnabarinus; (Boisduval) Boudreaux, 1956; synonymy according to Dupont (1979).

Acarus haematodes; Boisduval, 1867: 88.

Tetranychus telarius haematodes; (Boisduval) Murray, 1877: 101; synonymy according to Smith \& Baker (1968). 
Acarus ferrugineus; Boisduval, 1867: 90.

Tetranychus ferrugineus; (Boisduval) Murray, 1877: 103; synonymy according to Pritchard \& Baker (1955).

Acarus vitis; Boisduval, 1867: 92.

Tetranychus vitis; (Boisduval) Murray, 1877: 103; synonymy according to Pritchard \& Baker (1955).

Distigmatus pilosus; Donnadieu, 1875: 118; synonymy according to Pritchard \& Baker (1955).

Tetranychus major; Donnadieu, 1875: 120; synonymy асcording to Pritchard \& Baker (1955).

Tetranychus piger; Donnadieu, 1875: 121; synonymy according to Pritchard \& Baker (1955).

Tetranychus minor; Donnadieu, 1875: 121; synonymy according to Pritchard \& Baker (1955).

Tetranychus longitarsus; Donnadieu, 1875: 122; synonymy according to Pritchard \& Baker (1955).

Tetranychus plumistoma; Donnadieu, 1875: 122; synonymy according to Pritchard \& Baker (1955).

Tetranychus fici; Murray, 1877: 107; synonymy according to Pritchard \& Baker (1955).

Tetranychus eriostemi; Murray, 1877: 109; synonymy according to Pritchard \& Baker (1955).

Tetranychus inaequalis; Targioni Tozzetti, 1878: 251; synonymy according to Pritchard \& Baker (1955).

Tetranychus bimaculatus; Harvey, 1892: 133; synonymy according to Pritchard \& Baker (1955).

Tetranychus altheae; von Hanstein, 1901: 74.

Epitetranychus altheae; (von Hanstein) Zacher, 1916: 23; synonymy according to Pritchard \& Baker (1955).

Epitetranychus hamatus; Zacher, 1916: 25; synonymy according to Pritchard \& Baker (1955).

Epitetranychus aequans; Zacher, 1916: 25; synonymy according to Pritchard \& Baker (1955).

Epitetranychus alceae; Oudemans, 1928b: 290; synonymy according to Pritchard \& Baker (1955).

Tetranychus reinwardtiae; Oudemans, 1930b: 170;

Epitetranychus reinwardtiae; (Oudemans) Oudemans, 1931a 194; synonymy according to Pritchard \& Baker (1955).

Epitetranychus caldarii; Oudemans, 1931a: 194.

Tetranychus caldarii; (Oudemans) Geijskes, 1939: 40; synonymy according to Pritchard \& Baker (1955).

Tetranychus fragariae; Oudemans, 1931a: 226; synonymy according to Pritchard \& Baker (1955).

Tetranychus fransseni; Oudemans, 1931b: 227; synonymy according to Pritchard \& Baker (1955).

Tetranychus aspidistrae; Oudemans, 1931c: 258; synonymy according to Pritchard \& Baker (1955).

Tetranychus choisyae; Oudemans, 1931d: 274; synonymy according to Pritchard \& Baker (1955).

Tetranychus stellariae; Oudemans, 1931d: 275; synonymy according to Pritchard \& Baker (1955).

Tetranychus violae; Oudemans, 1931d: 277; synonymy according to Pritchard \& Baker (1955).

Tetranychus manihoti; Oudemans, 1931d: 289; synonymy according to Pritchard \& Baker (1955).

Eotetranychus inexpectatus; Andre, 1933: 131; synonymy according to Pritchard \& Baker (1955).

Tetranychus dahliae; Oudemans, 1937: 1022; synonymy according to Pritchard \& Baker (1955).

Eotetranychus scabrisetus; Ugarov \& Nikolskii, 1937: 33; synonymy according to Pritchard \& Baker (1955).

Eotetranychus cucurbitacearum; Sayed, 1946a: 90; synonymy according to Pritchard \& Baker, 1955).

Tetranychus multisetis; McGregor, 1950: 294; synonymy according to Pritchard \& Baker (1955).

Tetranychus arabicus; Attiah, 1967; synonymy according to Meyer (1987).

Tetranychus aduncus; Flechtmann, 1967: 20; synonymy according to Flechtmann \& Baker (1970).

Tetranychus ricinus; Saba, 1973: 63; synonymy according to Meyer (1987).

Registers on Hevea: São Paulo: Olímpia (Bellini et al. 2005a), on $H$. brasiliensis.

Types: on nettle (Fleurya aestuans L., Urticaceae), Regensburg, Germany, type specimens probably lost .

Remarks: this is one of the most serious tetranychid pests; attacks cotton (Gossypium herbaceum L.), Manihot sp. (Euphorbiaceae) and bean (Phaseolus vulgaris L., Fabaceae) (Prichard \& Baker 1955); registered on 912 host plants (Bolland et al.1998); in rubber trees, however, it was found in low number (Bellini et al. 2005a).

\section{Tetranychus sp.}

Registers on Hevea: São Paulo: São Jose do Rio Preto (Demite \& Feres 2005), on H. brasiliensis.

Remarks: Bolland et al.(1998, pg 141) wrongly mentioned $H$. brasiliensis as host for the species Paraponychus corderoi (Baker \& Pritchard, 1962) (C.H.W. Flechtmann, personal communication).

\section{Tydeidae Kramer, 1877}

\section{Afrotydeus kenyensis (Baker, 1970)}

Tydeus (Afrotydeus) kenyensis Baker, 1970: 165.

Tydeus kenyensis; Feres, 2000: 163.

Afrotydeus kenyensis; André, 1980: 106.

Registers on Hevea: São Paulo: Macaubal; Mato Grosso: Itiquira (Feres 2000), on $H$. brasiliensis.

Types: on coffee (Coffea arabica L., Rubiaceae), Kenya, deposited in BMNH. 


\section{Homeopronematus sp.}

Registers on Hevea: São Paulo: Olímpia (Bellini et al. 2005a), São Jose do Rio Preto (Demite \& Feres 2005), Cedral (Hernandes \& Feres 2006b); Goiás: Goianésia, on $H$. brasiliensis.

\section{Lorryia formosa Cooreman, 1958}

Lorryia formosa Cooreman, 1958: 6; Baker, 1968a: 995.

Registers on Hevea: São Paulo: Cedral (Feres et al. 2002, (Hernandes \& Feres 2006b), Olímpia (Bellini et al. 2005a), Pariquera-Açu (Zacarias \& Moraes 2002), São Jose do Rio Preto (Demite \& Feres 2005), Ibitinga; Mato Grosso: Itiquira (Feres 2000, Ferla \& Moraes 2002a), Pontes e Lacerda (Ferla \& Moraes 2002a), on H. brasiliensis.

Types: on Citrus sp. (Rutacea), Rhab, Morocco, deposited in IRSN.

Remarks: once considered harmful to citrus crops (Smirnoff 1957); collected from many host plants (Flechtmann 1973); exhibited reproduction by thelytoky when reared on rubber tree leaves, which is possibly the first case of thelytoky in Tydeoidea (Hernandes et al. 2006); collected in large population at the base of the leaves.

\section{Lorryia spp.}

Registers on Hevea: São Paulo: Cedral (Hernandes \& Feres 2006b), Olímpia (Bellini et al. 2005a), Pariquera-Açu (Zacarias \& Moraes 2002), Piracicaba (Zacarias \& Moraes 2002, Vis et al. 2006), Rio Claro (Flechtmann \& Arleu 1984); Mato Grosso: Itiquira, Pontes e Lacerda (Ferla \& Moraes 2002a), on $H$. brasiliensis.

Remarks: it may be more than one species; specimens from Rio Claro, Itiquira and Pontes e Lacerda were not examined.

\section{Melissotydeus sp.}

Registers on Hevea: São Paulo: Olímpia (Bellini et al. 2005a), on $H$. brasiliensis.

\section{Neolorryia boycei (Baker, 1944)}

Retetydeus boycei Baker, 1944: 78.

Lorryia boycei; Baker, 1968a: 1004; Feres, 2000: 163.

Neolorryia boycei; André, 1980: 127; Kazmierski, 1998: 350. Registers on Hevea: São Paulo: Reginópolis (Feres 2000), on $H$. brasiliensis.

Types: on moss, Laguna de Zempoala, Morelos, Mexico, deposited in USNM.

\section{Neolorryia sp.}

Registers on Hevea: São Paulo: Piracicaba (Vis et al. 2006), on $H$. brasiliensis.

\section{Parapronematus acaciae Baker, 1965}

Parapronematus acaciae Baker, 1965: 116.

Registers on Hevea: São Paulo: Pariquera-Açu (Zacarias \& Moraes 2002), Piracicaba (Zacarias \& Moraes 2002, Vis et al. 2006), on $H$. brasiliensis.

Types: on Acacia sp. leaf (Fabaceae), Leopoldville, Belgian Congo, deposited in USNM.

\section{Parapronematus spp.}

Registers on Hevea: São Paulo: José Bonifácio, Macaubal, Barretos (Feres 2000), Olímpia (Bellini et al. 2005a); Mato Grosso: Itiquira, Pontes e Lacerda (Ferla \& Moraes 2002a), on $H$. brasiliensis.

Remarks: it may be more than one species; specimens from Pontes e Lacerda were not examined.

\section{Pausia sp.}

Registers on Hevea: São Paulo: Piracicaba (Vis et al. 2006), on $H$. brasiliensis.

\section{Pretydeus curiosa (Ueckermann \& Smith-Meyer, 1979)}

Lorryia curiosa Ueckermann \& Smith-Meyer, 1979: 44.

Pretydeus curiosa; André, 1980: 143-144.

Registers on Hevea: São Paulo: Pariquera-Açu (Zacarias \& Moraes 2002), on H. brasiliensis.

Types: on Maytenus nemorosa (Eckl. Zeyh.) Marais (Celastraceae), Matubatuba (Zululand), deposited in NCAPPRI.

\section{Pretydeus sp.}

Registers on Hevea: São Paulo: Pariquera-Açu (Zacarias \& Moraes 2002), Piracicaba (Vis et al. 2006), on H. brasiliensis.

\section{Pronematus ubiquitus (McGregor, 1932)}

Tydeus ubiquitus McGregor, 1932: 62.

Pronematus ubiquitus; Thor, 1933: 46; Baker, 1939: 273; Baker, 1946: 255; Meyer \& Rodriguez, 1966: 19; Baker, 1968b: 1093.

Registers on Hevea: São Paulo: Piracicaba (Vis et al. 2006), on $H$. brasiliensis.

Types: on Citrus sp. foliage (Rutaceae) , Linsay, California, USA, deposited in USNM.

\section{Pronematus spp.}

Registers on Hevea: São Paulo: Cedral (Feres et al. 2002, Hernandes \& Feres 2006b), Pindorama, Taquaritinga (Feres et al. 2002), Ibitinga, Macaubal, Pindorama, Reginópolis (Feres 2000), Olímpia (Bellini et al. 2005a), Piracicaba 
(Zacarias \& Moraes 2002, Vis et al. 2006), São Jose do Rio Preto (Demite \& Feres 2005); Mato Grosso: Itiquira, Pontes e Lacerda (Ferla \& Moraes 2002a), on H. brasiliensis; Itiquira (Feres 2000), on H. benthamiana.

Remarks: Pronematus ubiquitus was reported as predator of eriophyids (Baker \& Wharton 1952); it may be more than one species; specimens from Itiquira and Pontes e Lacerda were not examined.

\section{Pseudolorryia cf. nicaraguensis}

Registers on Hevea: São Paulo: Piracicaba (Vis et al. 2006), on $H$. brasiliensis.

\section{Triophtydeus spp.}

Registers on Hevea: São Paulo: Piracicaba (Vis et al. 2006), Cedral, on $H$. brasiliensis.

\section{Tydeus (Tydeus) californicus (Banks, 1904)}

Tetranychoides californicus Banks, 1904: 54.

Tydeus californicus; Baker \& Wharton, 1952: 192; Fleschner \& Arakawa, 1953: 1092.

Tydeus (Tydeus) californicus; Baker, 1970: 174.

Registers on Hevea: São Paulo: Pariquera-Açu (Zacarias \& Moraes 2002), on H. brasiliensis.

Types: on orange leaves (Citrus sp, Rutaceae), Watsonville, California, U.S.A., institution of deposition not found.

\section{Tydeus (Tydeus) costensis Baker, 1970}

Tydeus (Tydeus) costensis Baker, 1970: 174.

Registers on Hevea: São Paulo: Pariquera-Açu (Zacarias \& Moraes 2002), on $H$. brasiliensis.

Types: on Datura sp. leaf (Solanacea), Birris, near Cartago, Costa Rica, deposited in USNM.

\section{Tydeus sp.}

Registers on Hevea: São Paulo: Monte Aprazível (Feres 2000); Campinas (Chiavegato 1968), on H. brasiliensis.

Remarks: in Campinas, it was collected in clones of rubber trees (Chiavegato 1968).

\section{ACARIDIDA}

\section{Acaridae Ewing \& Nesbitt, 1954}

\section{Caloglyphus sp.}

Registers on Hevea: São Paulo: São Jose do Rio Preto (Demite \& Feres 2005), on H. brasiliensis.

\section{Neotropacarus sp.}

Registers on Hevea: Mato Grosso: Itiquira (Ferla \& Moraes 2002a); São Paulo: Piracicaba (Zacarias \& Moraes 2002, Vis et al. 2006), Pariquera-Açu (Zacarias \& Moraes 2002), on $H$. brasiliensis.

\section{Tyrophagus putrescentiae (Schrank, 1781)}

Acarus putrescentiae Schrank, 1781: 521.

Tyrophagus putrescentiae; Oudemans, 1924: 250; Feres 2000: 169.

Registers on Hevea: São Paulo: Barretos, Buritama, Pindorama, Reginópolis; Mato Grosso: Itiquira (Feres 2000), Goiás: Goianésia, on H. brasiliensis.

Types: institution of deposition, host and locality types not found.

Remarks: pest of culture medium, insect food in laboratories and stored food (Flechtmann 1986).

\section{Tyrophagus sp.}

Registers on Hevea: Mato Grosso: Itiquira (Ferla \& Moraes 2002a), on H. brasiliensis.

\section{Histiostomatidae Hughs, 1976}

Unidentified species.

Registers on Hevea: São Paulo: Barretos, Buritama (Feres 2000), on H. brasiliensis.

Remarks: several species are found in wet environments, like exudations of wounded trees (Flechtmann 1975); only hypopus were collected.

\section{Winterschmidtiidae Oudemans, 1923}

\section{Czenspinskia sp.}

Registers on Hevea: São Paulo: Olímpia (Bellini et al. 2005a), Pariquera-Açu, Piracicaba (Zacarias \& Moraes 2002), São Jose do Rio Preto (Demite \& Feres 2005), Cedral (Hernandes \& Feres 2006b), on H. brasiliensis.

\section{Oulenzia sp.}

Registers on Hevea: São Paulo: Cedral (Feres et al. 2002, Hernandes \& Feres 2006b), Pariquera-Açu (Zacarias \& Moraes 2002), Pindorama (Feres et al. 2002), Olímpia (Bellini et al. 2005a), Piracicaba (Vis et al. 2006), São Jose do Rio Preto (Demite \& Feres 2005), Ibitinga; Mato Grosso: Itiquira (Feres 2000, Ferla \& Moraes 2002a), on H. brasiliensis.

Remarks: O. arboricola (Oudemans) was described from Hevea leaves in Sumatra; specimens were also collected on jute, in India; reported as phytophagous (Baker \& Wharton 1952). 


\section{ORIBATIDA}

\section{Oribatulidae Thor, 1929 \\ Spinoppia sp.}

Registers on Hevea: São Paulo: Piracicaba (Vis et al. 2006), on $H$. brasiliensis.

\section{Oripodidae Jacot, 1925 \\ Pirnodus sp.}

Registers on Hevea: São Paulo: Piracicaba (Vis et al. 2006), on $H$. brasiliensis.

Unidentified spp.

Registers on Hevea: São José do Rio Preto (Demite \& Feres 2005), Piracicaba (Vis et al. 2006).

Fifty four nominal species and about 50 unidentified species of mites were reported on rubber trees in Brazil. Calacarus heveae and Tenuipalpus heveae are economically important pests of that culture, reaching great populations at the end of the rainy season and beginning of the dry season. Phyllocoptruta seringueirae, being found in large populations in the State of Mato Grosso and São Paulo (Ferla \& Moraes 2002a, Bellini et al. 2005a, R.J.F. Feres, pers. comm.), is another eriophyid mite that deserves attention.

Seven of the nominal species of mites reported in rubber trees in Brazil belong to Tetranychidae, which comprises agricultural pests for several crops. Eutetranychus banksi was registered in great abundance in several rubber tree crops, although no evident damage to the leaves has been noticed.

The family with greatest number of species was Phytoseiidae (257), with preponderantly predatory species (McMurtry \& Croft 1997). Zacarias \& Moraes (2001) reported nine phytoseiid species on rubber trees in the southern region of the State of São Paulo, suggesting they could be helping to maintain low the population levels of some mite pests. Euseius citrifolius was the phytoseiid most commonly found on rubber trees of São Paulo State.

Stigmaeidae is another family with very abundant predatory species, with at least ten species reported to date. Ferla \& Moraes (2003b) observed high oviposition rate of Agistemus floridanus fed on C. heveae and T. heveae. However, due to the explosive populational increase and high abundance of these phytophagous in natural conditions, it is highly unlikely that A. floridanus can reduce significantly their population.

This work lists 31 sampling points in many States of Brazil (Figure 2). In most rubber tree plantations studied there were only a few isolated samplings (Baker 1945, Chiavegato 1968, Flechtmann \& Arleu 1984, Fazolin \& Pereira
1989, Flechtmann 1989, Vieira \& Gomes 1999, Feres 2000, 2001), but as soon as a harmful pest as $C$. heveae was discovered, several works arose in order to understand the seasonal occurrence of mites on that culture. In some rubber tree plantations of the State of São Paulo the authors conducted samplings every season of the year (Feres et al. 2002). A few studies in the States of São Paulo and Mato Grosso conducted monthly samplings for a year of analysis (Bellini et al. 2005, Ferla \& Moraes 2002). The study conducted in Cedral (Hernandes \& Feres 2006b), northwestern region of the State of São Paulo, represents the first long term study of mites of rubber trees, considering three years of monthly samplings, and provides many information concerning the seasonal occurrence of mites.

Not surprisingly, most surveys of mites in rubber trees in Brazil were made in the São Paulo State (Figure 2 ), which responds to up to $60 \%$ of the national latex yield (Gonçalves et al. 2001). That disparity in relation to the number of studies conducted in other Brazilian States also reflects the greater number of researchers working on mites in that state.

\section{Acknowledgements}

To agricultural engineer José F.C. Benesi (APABOR, S.J. Rio Preto, São Paulo, Brazil), for collecting and sending the material from Goianésia, Goiás; M.Sc. Rodrigo Damasco Daud (UNESP, S.J. do Rio Preto, São Paulo, Brazil), for the assistance in identification of the material from Goianésia; Prof. Dr. Carlos H.W. Flechtmann (ESALQ-USP, Piracicaba, São Paulo, Brazil), Prof. Dr. Carlos Amadeu L. Oliveira (UNESP, Jaboticabal, São Paulo, Brazil), for valuable suggestions on the manuscript; to Dr. Antônio Carlos Lofego (IB-USP), Ronald Ochoa, Debra D. Creel and Barry O’Connor (USNM Beltsville, Maryland, USA) for the valuable information concearning many holotype specimens. This work was partially supported by the State of São Paulo Research Foundation (FAPESP) within the BIOTA/FAPESP- Virtual Institute of Biodiversity Program (www.biota.org.br)Proc. $n^{\circ}$ 98/7099-0 and the National Council for Scientific and Technological Development (CNPq)Proc. $n^{\circ} 134081 / 2003-0$.

\section{References}

ANDRÉ, H.M. 1980. A generic revision of the family Tydeidae (Acari: Actinedida). IV-Generic descriptions, keys and conclusions. Bull. Ann. Soc. R. Belge Ent. 116:103-107.

ANDRÉ, M. 1933. Note sur “l’Araigneé rouge” dês serres du Muséum. Bull. Mus, Natl. Hist. Nat. Paris (ser.2). 5:302-308.

APONTE, O. \& McMURTRY, J.A. 1995. Revision of genus Iphiseiodes DeLeon (Acari: Phytoseiidae). Int. J. Acarol. 21:165-183. 
ARRUDA FILHO, G.P.de \& MORAES, G.J.de. 2003. Stigmaeidae mites (Acari: Raphgnathoidea) from Arecaceae of the Atlantic Forest in São Paulo State, Brazil. Neotrop. Entomol. 32:49-57.

ATTIAH, H.H. 1967c. Revision on the Tetranychus telarius complex in the U.A.R. with description of new species (Acarina: Tetranychidae). Bull. Soc. Entomol. Egypte. 51(7):7-10.

ATHIAS-HENRIOT, C. 1958. Contribution à la Connaissance du genre Typhlodromus Scheuten (Acariens, Parasitiformes, Phytoseiidae). Description de deux espèces nouvelles d'Algèrie et clé dês espèces du groupe finlandicus. Rev. Pathol. Veg. Entomol. Agric. 37:179-186.

BAKER, E.W. 1939. The fig mite, Eriophyes ficus Cotte, and other mites of the fig tree, Ficus carica Linn. Calif. Dep. Agr. 28(4):266-275.

BAKER, E.W. 1944. Tideidos Mexicanos (Acari, Tydeidae). Rev. Soc. Mex. Hist. Nat. 5:73-81.

BAKER, E.W. 1945. Mites of the genus Tenuipalpus (Acarina: Trichadenidae). Proc. Entomol. Soc. Wash., 47:333-344.

BAKER, E.W. 1946. Some Tydeidae from the fig tree (Ficus carica L.). Anales Escuela Nac. Cienc. Biol. Mex. 4(2-3):255-261.

BAKER, E.W. 1949a. A review of the mites of the family Cheyletidae in the United States National Museum. Proc. U.S. Natl. Mus. 99:267-320.

BAKER, E.W. 1949b. The genus Brevipalpus (Acarina:Pseudoleptidae). Am. Midl. Nat. 42:350-402.

BAKER, E.W. 1965. A review of the genera of the family Tydeidae (Acarina). In Advances of Acarology. 2:95-120. Cornell Univ. Press, Ithaca.

BAKER, E.W. 1968a. The genus Lorryia. Ann. Entomol. Soc. Am. 61:986-1008.

BAKER, E.W. 1968b. The genus Pronematus. Ann. Entomol. Soc. Am. 61:1091-1097.

BAKER, E.W. 1970. The genus Tydeus: Subgenera and species groups with descriptions of new species (Acarina:Tydeidae). Ann. Entomol. Soc. Am. 63:163-177.

BAKER, E.W. \& PRITCHARD, A.E. 1953. A review of the false spider mite genus Tenuipalpus Donnadieu (Acarina: Phytoptipalpidae). Ann. Entomol. Soc. Am. 46:317-336.

BAKER, E.W. \& PRITCHARD, A.E. 1960. The tetranychoid mites of Africa. Hilgardia. 29:455-574.

BAKER, E.W. \& PRITCHARD, A.E. 1962 (1963). Arañas rojas de America Central. Revta. Soc. Hist. Nat. 23:309-340.

BAKER, E.W. \& TUTTLE, D.M. 1987. The false spider mites of Mexico (Tenuipalpidae: Acari). Tech. Bull. / U.S. Dep. Agric. 1706:1-237.
BAKER, E.W., TUTTLE, D.M. \& ABBATIELLO, M.J. 1975. The false spider mites of northwestern and north central Mexico (Acarina:Tenuipalpidae). Smith. Contrib. Zool. 194:1-23.

BAKER, E.W. \& WHARTON, G.W. 1952. An introduction to acarology. The McMillan CO, New York.

BANKS, N. 1904. Class III, Arachnida, Order I, Acarina, four new species of injurious mites. Jour. N.Y. Entomol. Soc. 12:53-56.

BANKS, N. 1917. New mites, mostly economic (Arach., Acar.). Ent. News. 28:193-199.

BEER, R.E. \& NUCIFORA, A. 1965. Revisione dei generi della famiglia Tarsonemidae (Acarina). Boll. Zool. Agric. Bachic. Ser. 2. 7:19-43.

BELLINI, M.R., MORAES, G.J. de \& FERES, R.J.F. 2005a. Ácaros (Acari) de dois sistemas comuns de cultivo de seringueira no noroeste do Estado de São Paulo. Neotrop. Entomol. 34:475-484.

BELLINI, M.R., MORAES, G.J. de \& R.J.F. FERES. 2005 b. Plantas de ocorrência espontânea como substrato alternativo para fitoseídeos (Acari: Phytoseiidae) em cultivos de seringueira (Hevea brasiliensis Muell. Arg., Euphorbiaceae). Rev. Bras. Zool. 22(1):35-42.

BLANCHARD, E. 1940. Tres acaros daninos para los cultivos argentinos. Rev. Fac. Agron. La Plata (ter ероса) 24: 11-18.

BOISDUVAL, A. 1867. Essay sur L'entomologie horticole. Paris.

BOLLAND, H.R., GUTIERREZ, J. \& FLECHTMANN, C.H.W. 1998. World catalog of the spider mite family (Acari: Tetranychidae). Brill, Leiden.

BOUDREAUX, H.B. 1956. New species of Tetranychid mites. Pan. Pac. Ent. 30:181-186.

BUOSI, R., FERES, R.J.F., OLIVEIRA, A.R., LOFEGO, A.C. \& HERNANDES, F.A. 2006. Ácaros plantícolas (Acari) da “Estação Ecológica de Paulo de Faria”, Estado de São Paulo, Brasil. Biota Neotropica 6:(no prelo)

CANO, Y. \& ALCACIO, D. 1886. Una nueva especie de Arana Mexicana, Tetranychus dugesii. Naturaleza. 7: 197-200.

CHAGAS, C.M. 1973. Associação do ácaro Brevipalpus phoenicis (Geijskes) à mancha anular do cafeeiro. Biológico. 39:229-232.

CHANT, D.A. 1955. Notes on the mites of the genus Typhlodromus Scheuten, 1857 (Acarina: Laelaptidae), with descriptions of the males of some species and the female of a new species. Can. Entomol. 87:496-503.

CHANT, D.A. 1959. Phytoseiid mites (Acarina: Phytoseiidae). Part I. Bionomics of seven species in southeastern England. Part II. A taxonomic review of the family Phytoseiidae, with descriptions of thirty-eight new species. Can. Entomol. Suppl. 12:166p. 
CHANT, D.A. \& BAKER, E.W. 1965. The Phytoseiidae (Acarina) of Central America. Mem. Entomol. Soc. Canada. 41:1-56.

CHANT, D.A. \& McMURTRY, J.A. 1994. A review of the subfamilies Phytoseiinae and Typhlodrominae (Acari: Phytoseiidae). Int. J. Acarol. 20:223-310.

CHANT, D.A. \& YOSHIDA-SHAUL, E. 1983. A world review of five similar species groups in the genus Typhlodromus Scheuten. Part II. The conspicuus and cornus groups (Acarina:Phytoseiidae). Can. J. Zool. 61:1041-1057.

CHANT, D.A. \& YOSHIDA-SHAUL, E. 1984. A world review of the occidentalis species group in the genus Typhlodromus Scheuten (Acarina: Phytoseiidae). Can. J. Zool. 62:1860-1871.

CHANT, D.A. \& YOSHIDA-SHAUL, E. 1986. A new subfamily, Cydnodromellinae, in the family Phytoseiidae (Acarina: Gamasina). Can. J. Zool. 64:2811-2823.

CHIAVEGATO, LG. 1968. Contribuição para o conhecimento de alguns ácaros que ocorrem na seringueira (Hevea brasiliensis Muell. Arg.). I Reunião Anual Soc. Bras. Entomol. p. 67.

CHIAVEGATO, LG. 1980. Ácaros da cultura dos citros, p.469501. In: Rodriguez, O. \& F.C.P. Viégas (Ed.). Citricultura Brasileira. Fundação Cargill. Campinas. p.1-739.

CHILDERS, C.C., KITAJIMA, E.W., WELBOURN, W.C., RIVERA, C. \& OCHOA, R. 2001. Brevipalpus mites on citrus and their status as vectors of citrus leprosis. Manejo Integr. Plagas. 60:66-70.

COOREMAN, J. 1958. Notes et observations sur les Acariens. VII- Photia graeca n.sp. (Acaridia, Canestriniidae) et Lorryia formosa n. sp. (Stomatostigmata, Tydeydae). Bull. Inst. R. Sci. Nat. Belg. Sci. Entomol. 34:1-10.

DAUD, R.D. \& FERES, R.J.F. 2005. Diversidade e flutuação populacional de ácaros (Acari) em Mabea fistulifera Mart. (Euphorbiaceae) de dois fragmentos de mata estacional semidecídua em São José do Rio Preto, São Paulo, Brasil. Neotrop. Entomol. 34 (2):191-201.

DELEON, D. 1957. Three new Typhlodromus from southern Florida (Acarina: Phytoseiidae). Fla. Entomol. 40:141-144.

DELEON, D. 1958. Four new Typhlodromus from southern Florida (Acarina: Phytoseiidae). Fla. Entomol. 41:73-76.

DELEON, D. 1961. The genus Brevipalpus in Mexico. Part II (Acarina:Tenuipalpidae). Fla. Entomol. 44:41-52.

DELEON, D. 1962. Twenty-three new Phytoseiidae, mostly from southeastern United States (Acarina: Phytoseiidae). Fla. Entomol. 45:11-27.

DELEON, D. 1966. Phytoseiidae of British Guyana with keys to species (Acarina: Mesostigmata). Studies the Fauna of Suriname and other Guyanas, 8: 81-102.
DELEON, D. 1967. Some mites of the Caribbean Area. Part I. Acarina on plants in Trinidad, West Indies. Allen Press Inc., Lawrence.

DEMITE, P.R. \& FERES, R.J.F. 2005. Influência de vegetacão vizinha na distribuicão de acaros em seringal (Hevea brasiliensis Muell. Arg., Euphorbiaceae) em São Jose do Rio Preto, SP. Neotrop. Entomol. 34(5):829-836.

DENMARK, H. A. 1982. Revision of Galendromus Muma, 1961 (Acarina: Phytoseiidae). Int. J. Acarol. 8:133-167.

DENMARK, H.A. \& MUMA, M.H. 1970. Some phytoseiid mites of Paraguay (Phytoseiidae: Acarina). Fla. Entomol. 53:219-227.

DENMARK, H.A. \& MUMA, M.H. 1972. Some Phytoseiidae of the Colombia (Acarina : Phytoseiidae). Fla. Entomol. 55:19-29.

DENMARK, H.A. \& MUMA, M.H. 1973. Phytoseiid mites of Brazil (Acarina: Phytoseiidae). Rev. Bras. Biol. 33:235-276.

DENMARK, H.A. \& MUMA, M.H. 1989. A revision of the genus Amblyseius Berlese, 1914 (Acari: Phytoseiidae). Occ. Pap. Florida St. Coll. Arthropods 4:1-149.

DONNADIEU, A.L. 1875. Recherches pour servir à l'historie dês Tétranyques. Ann. Soc. Lyon. 12:1-134.

DUGES, A. 1834. Recherches sur l’orde des Acariesn em general at le famille des Trombidies in particular. An. Sci. Nat. Paris (ser. 2) 1:5-46.

DUPONT, L.M. 1979. On gene flow between Tetranychus urticae Koch, 1836 and Tetranychus cinnabarinus (Boisduval) Boudreaux, 1956 (Acari: Tetranychidae): synonomy between the two species. Entomol. Exp.Appl. 25:297-303.

EHARA, S. 1993. Two new species of the genus Agistemus Summers from Malasya (Acari, Stigmaeidae). Jour. Acarol. Soc. Jpn. 2:79-82.

EL-BENHAWY, E.M. 1984. Description of some Phytoseiidae mites from Brazil (Acarina: Phytoseiidae). Acarologia 25:125-144.

ESTEBANES, \& BAKER, E.W. 1968. Aranas rojas de Mexico (Acarina: Tetranychidae). Ann. Esc. Nac. Mex. 15:61-133.

EWING, H. E. 1939. A revision of the mites of the subfamily Tarsoneminae of North America, the West Indies and Hawaiian Islands. Tech. Bull. / U.S. Dep. Agric. (653):1-63.

FAIN, A., BOCHKOV,A.V. \& CORPUZ-RARUS, L.A. 2002. A revision of the Hemicheyletia generic group (Acari: Cheyletidae). Bull. Inst. Roy. Sci. Nat. 72:27-66.

FARIAS, A.R., FLECHTMANN, C.H.W., MORAES, G.J. de \& McMURTRY, J.A. 1981. Predadores do ácaro verde da mandioca, no nordeste do Brasil. Pesqui. Agropecu. Bras. 16:313-317. 
FAZOLIN, M. \& PEREIRA, L.V. 1989. Ocorrência de Oligonychus gossypii (Zacher 1921) (Acari:Tetranychidae) em seringueiras cultivadas. An. Soc. Entomol. Bras. 18:199-202.

FERES, R.J.F. 1992. Anew species of Calacarus Keifer (Acari, Eriophyidae, Phyllocoptinae) from Hevea brasiliensis Muell. Arg. (Euphorbiaceae) from Brazil. Int. J. Acarol. 18:61-65.

FERES, R.J.F. 1998. Two new Phyllocoptinae mite (Acari: Eriophyidae) from Hevea brasiliensis Muell. Arg. (Euphorbiaceae) from Brazil. Int. J. Acarol. 24:69-74.

FERES, R.J.F. 2000. Levantamento e observações naturalísticas da acarofauna (Acari, Arachnida) de seringueiras cultivadas (Hevea spp., Euphorbiaceae) no Brasil. Rev. Bras. Zool. 17: 157-173.

FERES, R.J.F. 2001a. Primeiro registro de ácaros eriofiídeos (Acari, Eriophyidae) em seringueiras (Hevea brasiliensis Muell. Arg., Euphorbiaceae) da Floresta Amazônica, Brasil. Rev. Bras. Zool. 18:343-345.

FERES, R.J.F. 2001b. Ácaros eriofiídeos (Acari, Eriophyidae) em seringueira (Hevea brasiliensis Muell. Arg., Euphorbiaceae) no Brasil, p.31-36, In Sa, L.A.N. \& Moraes, G.J. (orgs). Ácaros de importância quarentenária. Embrapa Meio Ambiente, Jaguariúna.

FERES, R.J.F. \& MORAES, G.J. de. 1998. Phytoseiid mites (Acari: Phytoseiidae) from wood areas in the State of São Paulo, Brazil. Syst. Appl. Acarol. 3:125-132.

FERES, R.J.F. \& NUNES, M.A. 2001. Ácaros (Acari, Arachnida) associados a euforbiáceas nativas em áreas de cultivo de seringueiras (Hevea brasiliensis Muell. Arg., Euphorbiaceae) na região noroeste do Estado de São Paulo, Brasil. Rev. Bras. Zool. 18:1253-1264.

FERES, R.J.F., ROSSA-FERES, D. de C., DAUD, R.D. \& SANTOS, R.S. 2002. Diversidade de ácaros (Acari, Arachnida) em seringueiras (Hevea brasiliensis Muell. Arg., Euphorbiaceae) na região noroeste do Estado de São Paulo, Brasil. Rev. Bras. Zool. 19:137-144.

FERLA, N.J. \& MORAES, G.J. de. 2002a. Ácaros (Arachnida, Acari) da seringueira (Hevea brasiliensis Muell. Arg.) no Estado do Mato Grosso, Brasil. Rev. Bras. Zool. 19:867-888.

FERLA, N.J. \& MORAES, G.J. de. 2002b. Ácaros predadores (Acari) em plantas nativas e cultivadas do Estado do Rio Grande do Sul, Brasil. Rev. Bras. Zool. 19:1011-1031.

FERLA, N.J. \& MORAES, G.J. de. 2003a. Ciclo biológico de Calacarus heveae Feres, 1992 (Acari, Eriophyidae). Rev. Bras. Entomol. 47:399-402.

FERLA, N.J. \& MORAES, G.J. de. 2003b. Oviposição dos ácaros predadores Agistemus floridanus Gonzalez, Euseius concordis (Chant) e Neoseiulus anonymus (Chant \& Baker) (Acari) em resposta a diferentes tipos de alimentos. Rev. Bras. Zool. 20:153-155.
FLECHTMANN, C.H.W. 1967. Contribuição para o conhecimento dos ácaros de plantas de algumas regiões do estado de São Paulo. Tese de Doutoramento, ESALQUSP, Piracicaba. p.1-47.

FLECHTMANN, C.H.W. 1973. Lorryia formosa Cooreman, 1958 - Um ácaro dos citros pouco conhecido no Brasil. Cienc. Cult. 25:1179-1181.

FLECHTMANN, C.H.W. 1975. Elementos de Acarologia. Livraria Nobel S.A, São Paulo.

FLECHTMANN, C.H.W. 1979. Tuckerella ornata (Tucker), um ácaro novo para o Brasil e outros Tetranychoidea (Acari) do Estado do Pará. Na. Esc. Sup. Agric. "Luiz de Queiroz”, Piracicaba. 36:615-620.

FLECHTMANN, C.H.W. 1986. Ácaros em produtos armazenados e na poeira domiciliary. FEALQ, Piracicaba.

FLECHTMANN, C.H.W. 1989. Seringueira (Hevea sp.) um novo hospedeiro para Oligonychus gossypii (Zacher, 1921) (Acari: Tetranychidae). Bol. Mus. Emílio Goeldi, Ser. Zool. 5: 27-128.

FLECHTMANN, C.H.W. \& ABREU, J.M. 1973. Ácaros fitófagos do Estado da Bahia, Brasil. Cienc. Cult. $25: 244-251$.

FLECHTMANN, C.H.W. \&ARLEU, R.J. 1984. Oligonychus coffeae (Nietner, 1981), um ácaro tetraniquídeo da seringueira (Hevea brasiliensis) novo para o Brasil e observações sobre outros ácaros desta planta. Ecossistema 9:123-125.

FLECHTMANN, C.H.W. \& BAKER, E.W. 1970. A preliminary report on the Tetranychidae (Acarina) of Brazil. Ann. Entomol. Soc. Am. 63:156-163.

FLECHTMANN, C.H.W. \& BAKER, E.W. 1975. A report on the Tetranychidae (Acari) of Brazil. Rev. Bras. Entomol. 19:111-122.

FLESCHNER, C.A. \&ARAKAWA, K.Y. 1953. The mite Tydeus californicus on citrus and avocado leaves. J. Econ. Entomol. 45(6):1092

FOURCROY, A.F. de. 1785. Entomolgia Parisiensis, 1:1-231; 2:233-544.

GARMAN, P. 1958. New species belonging to the genera Amblyseius and Amblyseiopsis with keys to Amblyseius, Amblyseiopsis and Phytoseiulus. Ann. Ent. Soc. Am. 51:69-79.

GEIJSKES, D.C. 1939. Beitraege zur Kenntnis der europeaischen Spinnmilben (Acari, Tetranychidae), mit besonderer Beruecksichtigung der niederlaendischen Arten. Meded. Landbouwhoogesch. Wagening. 42:1-68.

GONÇALVES, P.S., BATAGLIA, O.C., ORTOLANI, A.A. \& FONSECA. F.S. 2001. Manual de heveicultura para o Estado de São Paulo. Boletim Técnico IAC. 189. Campinas. 
GONDIM Jr., M.G.C. \& MORAES, G.J. de. 2001. Phytoseiid mites (Acari: Phytoseiidae) associated with palm trees (Arecaceae) in Brazil. Syst. Appl. Acarol. 6:65-94.

GONZALEZ-RODRIGUEZ, H. 1965. A taxonomic study of the genera Mediolata, Zetzellia and Agistemus (Acarina: Stigmaeidae). Univ. Calif. Public. Entomol. 41:1-64.

GONZALEZ-RODRIGUEZ, H. 1975. Revision of the Brevipalpus phoenicis "complex", with descriptions of new species from Chile and Thailand (Acarina, Tenuipalpidae). Acarologia 17:82-91.

GUTIERREZ, J. 1968. Tetranychidae nouveaux de Madagascar (Quatrième note). Acarologia. 10:1-28.

HANSTEIN, R.V. 1901. Beitrage zur Kenntnis der Gattung Tetranychus Duf. Zts. Wiss. Zool. 70:58-108.

HARVEY, F.L. 1892. The two-spotted mite. Ann. Rept. Maine Agric. Exp. Sta. 133-144.

HERNANDES, F.A. \& R.J.F. FERES. 2005a. Two new species of Zetzellia Oudemans (Acari: Stigmaeidae) that threaten the concept of genera: disgeneric marriage? Zootaxa. 1048:27-44.

HERNANDES, F.A. \& R.J.F. FERES. 2006a. Tetrabdella neotropica (Acari: Bdellidae), new genus and species from Brazil. Zootaxa. 1135:57-68.

HERNANDES, F.A. \& R.J.F. FERES. 2006b. Diversidade e sazonalidade de ácaros (Acari) em seringal (Hevea brasiliensis Muell. Arg.) na regiao Noroeste do Estado de São Paulo. Neotrop. Entomol. 35 (in press).

HERNANDES, F.A., FERES, R.J.F. \& NOMURA, F. 2006. Biological cycle of Lorryia formosa Cooreman, 1958 (Acari: Tydeidae) in rubber tree leaves. Exp. Appl. Acarol. (in press)

HIRSCHMANN, W. 1962. Gangsystematik der Parasitiformes. Acarol. Schrift. Verg. Milb., HirschmannVerlag, Furth/Bay. 5:1-80.

HIRST, S. 1923. On some new or little-known species of Acari. Proc. Zool. Soc. Lond. 1923:971-1000.

HIRST, S. 1926. Descriptions of new mites including four new species of “red spider”. Proc. Zool. Soc. London. 1926:825-841.

KALISZEWSKI, M. 1993. Key to Paleartic females of the genus Tarsonemus. Wydawnictwo Naukowe Uniwersytet Im. Adama Mickiewica w Poznaniu, Poznan, Seria Zoologia 14:1-204.

KAZMIERSKI, A. 1998. Tydeidae of the world: generic relationships, new and redescribed taxa and keys to all species. A revision of the subfamilies Pretydeinae and Tydeinae (Acari: Actinedida: Tydeidae) - part 4. Acta Zool. Cracov. 41:283-455.

KLEIN, H.Z. 1936. Contributions to the knowledge of the red spiders in Palestine. Bull. Agr. Sta. Rehoroth. 21:1-63.
KOCH, C.L. 1836. Deutsche Crustacea, Myriapoda, Arachnida. Fasc. 1.

KOCH, C.L. 1838. Deutsche Crustacea, Myriapoda, Arachnida. Fasc. 17.

KOCH, C.L. 1842. Uebersicht des Arachnidensystems, 3:1131, Nurnberg, Germany.

KREITER, S. \& MORAES, G.J. de. 1997. Phytoseiid mites (Acari: Phytoseiidae) from Guadeloupe and Martinique. Fla. Entomol. 80:376-382.

LINNAEUS, C. 1758. Systema Naturae, Ed. X. (Systema naturae per regna tria naturae, secundum classes, ordines, genera, species, cum characteribus, differentiis, synonymis, locis. Tomus I. Editio decima, reformata.) Holmiae. Systema Nat. ed. 10 i-ii + 1-824.

LOFEGO, A.C., MORAES, G.J. de \& McMURTRY, J.A. 2000. Three new species of phytoseiid mites (Acari: Phytoseiidae) from Brazil. An. Soc. Entomol. Bras. 29:461-467.

MATIOLI,A.L., UECKERMANN, E.A. \& OLIVEIRA, C.A.L. de. 2002. Some Stigmaeid and Eupalopsellid mites from citrus orchards in Brazil (Acari: Stigmaeidae and Eupalopsellidae). Int. J. Acarol. 28:99-120.

McGREGOR, E.A. 1914. Four new tetranychids. Ann. Entomol. Soc. Am. 7: 354-364.

McGREGOR, E.A. 1917. Descriptions of seven new species of red spiders. Proc. U.S. Natl. Mus. 51:581-590.

McGREGOR, E.A. 1919. The red spiders of America and a few European species likely to be introduced. Proc. U.S. Nat. Mus. 56: 641-679.

McGREGOR, E.A. 1932. The ubiquitous mite, a new species on citrus. Proc. Entomol. Soc. Wash. 34:60-63.

McGREGOR, E.A. 1935. The Texas citrus mite, a new species. Proc. Ent. Soc. Wash. 37:161-165.

McGREGOR, E.A. 1950. Mites of the family Tetranychidae. Am. Midl. Nat. 44:257-420.

McMURTRY, J.A. 1983. Phytoseiidae mites from Guatemala, with descriptions of two new species and redefinitions of the genera Euseius, Typhloseiopsis, and Typhlodromus occidentalis species group (Acari: Mesostigmata). Int. J. Acarol. 25:249-272.

McMURTRY J.A. \& MORAES, G.J. de. 1989. Some phytoseiid mites from Peru with descriptions of four new species (Acari: Phytoseiidae). Int. J. Acarol. 15:179-188.

McMURTRY, J.A. \& CROFT, B.A. 1997. Life-styles of phytoseiid mites and their roles in biological control. Annu. Rev. Entomol. 42:291-321.

McMURTRY, J.A. \& HUFFAKER, C.B. \& DRIE, Van De. 1970. Ecology of tetranychid mites and their natural enemies: a review. 1. Tetranychid enemies: their biological characters and the impact of spray practices. Hilgardia. 40:331-390. 
MEYER, M.K.P.S. 1974. A revision of the Tetranychidae of Africa (Acari) with a key to the genera of the world. Entomology Mem. Dep. agric. tech. Serv. Repub. S. Afr. 36:1-291.

MEYER, M.K.P.S. 1979. The Tenuipalpidae (Acari) of Africa with keys to the world fauna. Entomology Mem. Dep. agric. tech. Serv. Repub. S. Afr. 50:1-135.

MEYER, M.K.P.S. 1987. African Tetranychidae (Acari: Prostigmata), with reference to the world genera. Entomology Mem. Dep. Agric. Wat. Supply Repub. S. Afr. 69:1-175.

MEYER, M.K.P.S. \& RODRIGUES, M. da C. 1965. Acari associated with cotton in Southern Africa (with reference to other plants). Garcia Orta. Ser. Zool. 13:195-226.

MEYER, M.K.P.S. \& UECKERMANN, E.A. 1989. African Raphgnathoidea (Acari: Prostigmata). Tech. Commun./ Dep. Agric. Water Supply, Repub. S. Afr. 74:1-58.

MORAES, G.J. de, ALENCAR, J.A. \& WENZEL NETO, F. 1988. Exploitations for natural enemies of the cassava green mite in Brazil. In: Proc. Symp. Int. Soc. Trop. Root Crops $8^{\text {th }}: 351-353$.

MORAES, G.J. de, DENMARK, H.A. \& GUERRERO, J.M. 1982. Phytoseiid mites of Colombia (Acarina: Phytoseiidae). Int. J. Acarol. 8:15-22.

MORAES, G.J de, KREITER, S. \& LOFEGO,A.C. 1999 (2000). Plant mites (Acari) of the French Antilles. 3. Phytoseiidae (Gamasida). Acarologia 40:237-264.

MORAES, G.J. de \& McMURTRY, J.A. 1983. Phytoseiid mites (Acarina) of northeastern Brazil with descriptions of four new species. Int. J. Acarol. 9:131-148.

MORAES, G.J. de, McMURTRY, J.A. \& DENMARK, H.A. 1986. A catalog of the mite family Phytoseiidade. References to taxonomy, synonymy, distribution and habitat. EMBRAPA-DDT, Brasília.

MORAES, G.J. de, McMURTRY, J.A. \& DENMARK, H.A. 2004. A revised catalog of the mite family Phytoseiidae. Zootaxa. 434: 1-494.

MORAES, G.J. de, \& MESA, N.C. 1988. Mites of the family Phytoseiidae (Acari) in Colombia, with descriptions of three new species. Int. J. Acarol. 14:71-88.

MORAES, G.J. de, MESA, N.C. \& BRAUN, A. 1991. Some phytoseiid mites of Latin America (Acari: Phytoseiidae). Int. J. Acarol. 17:117-139.

MORAES, G.J. de \& OLIVEIRA, J.V. 1982. Phytoseiid mites of coastal Pernambuco in northeastern Brazil. Acarologia 23:315-318.

MUMA, M.H. 1961. Subfamilies, genera, and species of Phytoseiidae (Acarina: Mesostigmata). Bull. Florida St. Mus. Biol. Sci. 5:267-302.

MUMA, M.H. 1963. The genus Galendromus Muma, 1961 (Acarina: Phytoseiidae). Fla. Entomol. 1:15-41.
MUMA, M.H. 1964. Cheyletidae (Acarina: Trombidiformes) associated with citrus in Florida. Fla. Entomol. 47:239-253.

MUMA, M.H. \& DENMARK, H.A. 1968. Some generic descriptions and name changes in the family Phytoseiidae (Acarina: Mesostigmata). Fla. Entomol. 51:229-240

MUMA, M.H., DENMARK, H.A. \& DELEON, D. 1970. Phytoseiidade of the Florida. Arthropods of Florida and neighboring land areas, 6. Florida Department of Agriculture and Consume Services, Division of Plant Industry, Gainesville.

MURRAY, A. 1877. Economic Entomology, Aptera. Chapman \& Hall. London.

NESBITT, J. 1951. A taxonomic study of the Phytoseiinae (Family Laelapidae) Predaceous upon Tetranychidae of economic importance. Zool. Verhandei. 12:1-64.

NIETNER, J. 1861. Observations on the enemies of the coffee tree in Ceylon. Ceylon, $31 \mathrm{p}$.

OOMEN, P.A. 1982. Studies on population dynamics of the scarlet mite, Brevipalpus phoenicis, a pest of tea in Indonesia. Medelingen Landbouwhogeschool. 82:1-88.

OUDEMANS, A.C. 1924. Acarologische Aanteekeningen. 74. Entomol. Ber. Amst. 6:249-260.

OUDEMANS, A.C. 1928. Acarologische Aanteekeningen. 89. Entomol. Ber. Amst. 7:285-293.

OUDEMANS, A.C. 1929. Acarologische Aanteekeningen. 98. Entomol. Ber. Amst. 7:476-485.

OUDEMANS, A.C. 1930. Acarologische Aanteekeningen. 105. Entomol. Ber. Amst. 8:157-172.

OUDEMANS, A.C. 1931a. Acarologische Aanteekeningen. 106. Entomol. Ber. Amst. 8:189-204.

OUDEMANS, A.C. 1931b. Acarologische Aanteekeningen. 107. Entomol. Ber. Amst. 8:221-236.

OUDEMANS, A.C. 1931c. Acarologische Aanteekeningen. 108. Entomol. Ber. Amst. 8:251-263.

OUDEMANS, A.C. 1931d. Acarologische Aanteekeningen. 109. Entomol. Ber. Amst. 8:272-280.

OUDEMANS, A.C. 1937. Kritisch Historich Overzicht der Acarologie. 3 C. 1:1-1348.

PONTIER, K.J; FLECHTMANN, C.H.W. 1999. Description of male Tenuipalpus heveae Baker, 1945 (Acari, Prostigmata, Tenuipalpidae). Int. J. Acarol. 25(4):293-296.

PONTIER, K.J; FLECHTMANN, C.H.W. 2000. Description of the immature stages of Tenuipalpus heveae Baker, 1945 (Acari, Prostigmata, Tenuipalpidae). Syst. Appl. Acarol. 5:77-81.

PONTIER, K.J.B., MORAES, G.J. de \& KREITER, S. 2000. Biology of Tenuipalpus heveae (Acari, Tenuipalpidae) on rubber tree leaves. Acarologia. 41:423-427. 
PRITCHARD, A.E. \& BAKER, E.W. 1955. A revision of the spider mite family Tetranychidae. Pacific Coast Entomological Society. Memoirs series vol.2, San Francisco.

PRITCHARD, A.E. \& BAKER, E.W. 1958. The false spider mites (Acarina: Tenuipalpidae). Univ. Calif. Publ. Entomol. 14:175-274.

RAHAMAN, K.A. \& SAPRA, A.N. 1940. Mites of the family Tetranychidae from Lyallpur with descriptions of four new species. Proc. Ind. Acad. Sci. (ser. B). 11:177-196.

RAKHA, M.A. \& McCOY, 1984. Eupalopsellid mites on Florida citrus, with a description of Exothorhis caudata Summers development stages (Eupalopsellidae: Raphnathoidea). Fla. Entomol. 68(1):141-144.

REIS, P.R., CHIAVEGATO, L.G., ALVES, E.B. \& SOUZA, E.O. 2000. Ácaros da família Phytoseiidae associados aos citros no município de Lavras, sul de Minas Gerais. An. Soc. Entomol. Brasil. 29:95-104.

RIMANDO, L.C. \& CORPUZ-RAROS, L.A. 1996. Some Philipine Raphgnathoidea (Acari) - I. Genera Paraeupalopsellus and Exothorhis (Eupalopsellidae). Phil. Entomol. 10(2):97-117.

RODRIGUES, M. Da C. 1968. Acarina de Moçambique Catálogo das espécies relacionadas com a agricultura. Agron. Moçamb. 2:215-256.

SABA, F. 1973. Les acariens nuisibles aux plantes cultivees au Moroc. Al Awamia. 49(3):69-97.

SATO, M.E., RAGA, A., CERÁVOLO, L.C., ROSSI, A.C. \& POTENZA, M.R. 1994. Ácaros predadores em pomar cítrico de Presidente Prudente, Estado de São Paulo. An. Soc. Entomol. Bras. 23:435-441.

SAYED, M.T. 1946a. Description of Tenuipalpus granati nov. spec. and Brevipalpus pyri nov. spec. $1^{\mathrm{er}}$ Ent. Bull. Soc. Fouad. $1^{\text {er } 30: 99-104 . ~}$

SAYED, M.T. 1946b. Contribution to the knowledge of the Acarina of Egypt: V-Five new species of Tetranychidae. Bull. Soc. Fouad. ${ }^{\text {er }}$ Ent. 30:79-97.

SAYED, M.T. 1946c. The genus Anychus McGregor in Egypt and the Sudan. Bull. Soc. Fouad. I ${ }^{\text {er }}$ Ent. 30:143-148.

SCHICHA, E. \& ELSHAFIE, M. 1980. Four new species of phytoseiid mites from Austrália, and three species from América redescribed (Acari: Phytoseiidae). Jour. Aust. Entomol. Soc. 19:27-36.

SCHRANK, F. von de P. 1781. Enumeratio Insectorum Austriae Indigenorum. Ausgutae Vindelicorum. Augsburg.

SMILEY, R. L. 1969. Further studies on the Tarsonemidae, II (Acarina). Proc. Entomol. Soc. Wash. 71:218-229.

SMILEY, R.L. 1967. Some Tarsonemidae of the Republic of the Congo (Acarina). Proc. Entomol. Soc. Wash. 66:145-150.
SMIRNOFF, W.A. 1957. An undescribed species of Lorryia (Acarina, Tydeidae) causing injury to citrus trees in Morocco. J. Econ. Entomol. 50:36-362.

SMITH, F.F. \& BAKER, E.W. 1968. Names of the two-spotted spider mite and the carmine spider mite to be redesigned. Co-op. Econ. Insect. Rep. USDA. 18:1-1080.

SUMMERS, F.F. 1960. Eupalopsis and eupalopsellid mites (Acarina: Stigmaeidae, Eupalopsellidae). Fla. Entomol. 43:119-138.

SUMMERS, F.M. \& D.W. PRICE. 1970. Revision of the family Cheyletidae. Univ. Calif. Publ. Entomol. 61:1-153.

SWIFT, S.F. 1997. First records of mites in the family Eupalopsellidae (Acari: Prostigmata: Raphignathoidea) in the Hawaiian Islands. Bishop Mu.Occ.Pap. 49:39-41.

TANZINI, M.R.; ALVES, S.B.; TAMAI, M.A.; MORAES, G.J. de; FERLA, N.J. 1999. An epizootic of Calacarus heveae (Acari: Eriophyidae) caused by Hirsutella thompsonii on rubber trees. Exp. Appl. Acarol. 24: 141-144.

TARGIONI TOZZETTI, A. 1878. Relazione intorno ai lavori della Stazione di Entomologia agraria di Firenze per l'anno 1876. Acaridei. Ann. Agr. 1:242-275.

THOR, S. 1933. Acarina Tydeidae, Ereynetidae. Tierreich. 60:1-82.

TUCKER, R.W.E. 1926. Some South Africa mites. Mainly Tetranychidae and Eriophyidae. Union S. Africa Dept. Agr. Div. Ent. Mem. 5:3-15.

UECKERMANN, E.A. \& SMITH-MEYER, M.K.P. 1979.African Tydeidae (Acari). I. The genus Lorryia Oudemans, 1925. Phytophylactica. 11:43-50.

UGAROV, A.A. \& NIKOLSKII, V.V. 1937. Systematic study of spider mites from Central Asia [in Russian]. Tr. SredneAziat. Stn. Zashch. Rast. 2: 26-64.

VAN der MERWE, G.G. 1968. A taxonomic study of the family Phytoseiidae (Acari) in South Africa with contributions to the biology of two species. S. Afr. Dep. Agric. Tec. Serv. Entomol. Mem. 18:1-198.

VIEIRA, M.R.; FABRI, E.G.; OLIVEIRA, E.A. 2000. Sintomatologia do ataque de Calacarus heveae em seringueira (Hevea brasiliensis). Rev. Agric. 75(3):405-414.

VIEIRA, M.R. \& GOMES, E.C. 1999. Sintomas, desfolhamento e controle de Calacarus heveae Feres, 1992 (Acari, Eriophyidae) em seringueira (Hevea brasiliensis Muell. Arg.). Cult. Agron. 8:39-52.

VIEIRA, M.R. \& GOMES, E.C. 2001. Avaliação de acaricidas no controle de Calacarus heveae Feres, 1992 (Acari: Eriophyidae) em seringueira através de contagem em campo. Cult. Agron. 10:145-158. 
VIS, R.D., MORAES, G.J.de \& BELLINI, M.R. 2006. Mites (Acari) of rubber trees (Hevea brasiliensis, Euphorbiaceae) in Piracicaba, State of São Paulo, Brazil. Neotrop. Entomol. 35: 112-120.

VOLGIN, V.I. 1955. In Paulovskii, E.N. Acarina of rodents of the USSR fauna. Akad. Nauk SSSR, Zool. Inst. Opredelitelipo faune. 59:1-459.

VOLGIN, V.I. 1969. Acarina of the family Cheyletidae of the World. Akad. Nauk. SSSR Zool. Inst. Opredelitelipo faune. 59:1-495.

WALLACE, M.M.H. \& MAHON, J.A. 1972. The taxonomy and biology of Australian Bdellidae (Acari). 1, Subfamilies Bdellinae, Spinibdellinae and Cytinae. Acarologia 14:544-580.

WOOD, TG. 1973. Revision of Stigmaeidae (Acari: Prostigmata) in the Berlese collection. Acarologia 15:76-95.

WOOD-MASON, J. 1884. Report on the tea-mite and teabug of Assam. Taylor \& Francis Pub. London.

WOOLLEY, T.A. 1988. Acarology: mites and human welfare. Library of Congress cataloging in Publication. Fort Collins, Colorado.

WRENSCH, D.L. \& EBBERT, M.A. 1993. Evolution and diversity of sex ratios in insects and mites. Chapman \& Hall. New York.

YOSHIDA-SHAUL, E. \& CHANT, D.A. 1997. A world review of the genus Phytoscutus Muma (Phytoseiidae: Acari). Acarologia. 38:219-238.

ZACARIAS, M.S. \& MORAES, G.J. de. 2001. Phytoseiid mites (Acari) associated with rubber trees and other euphorbiaceous plants in southeastern Brazil. Neotrop. Entomol. 30:579-586.

ZACARIAS, M.S. \& MORAES, G.J. de. 2002. Mite diversity (Arthropoda: Acari) on euphobiaceous plants in three localities in the State of São Paulo. Biota Neotropica. 2:1-12.

ZACHER, F. 1916. Zur Kenntnis der Spinnmilben. Mitt. Kais. Biol. Anst. Land-Forst. 16:19-25.

ZACHER, F. 1921. Neue und wenig bekannte Spinnmilben. Ztschr. Ang. Entomol. 7:181-187.
Title: Review about mites (Acari) of rubber trees (Hevea spp., Euphorbiaceae) in Brazil

Authors: Fábio Akashi Hernandes \& Reinaldo José Fazzio Feres

Biota Neotropica, Vol. 6 ( number 1): 2006

http://www.biotaneotropica.org.br/v6n1/pt/ abstract?article+bn00406012006

Date Received 06/15/2005 - Revised 12/05/2005

Accepted 01/01/2006

ISSN 1676-0611 


\section{INDEXES}

Species and families of mites found in rubber trees in Brazil:

(current synonyms in bold)

Acaridae

Acarus cinnabarinus

Acarus coffeae

Acarus putrescentiae

Acarus telarius

Aff. Acaphyllisa sp.

Aff. Aceria sp.

Aff. Chakrabartiella sp.

Afrotydeus kenyensis

Agistemus floridanus

Agistemus aff. floridanus

Agistemus sp.

Allonychus braziliensis

Amblyseiopsis ovatus

Amblyseius (Amblyseius) saopaulus

Amblyseius (Iphiseius) concordis

Amblyseius acalyphus

Amblyseius anonymus

Amblyseius compositus

Amblyseius concordis

Amblyseius dominigos

Amblyseius idaeus

Amblyseius impeltatus

Amblyseius neochiapensis

Amblyseius operculatus

Amblyseius ovatus

Amblyseius saopaulus

Amblyseius sexpilis

Amblyseius tunus

Amblyseius zuluagai

Anychus africanus

Anychus latus

Anychus? latus

Anychus banksi

Anychus clarki

Anychus orientalis

Anychus ricini

Anychus rusti

Anychus verganii

Atrichoproctus uncinatus

Bdellidae

Brevipalpus mcbridei

Brevipalpus papayensis

Brevipalpus phoenicis

Brevipalpus yothersi

Brevipalpus sp.

Calacarus heveae

Caloglyphus sp.

Cheletogenes sp.

Cheletomimus (Hemicheyletia) wellsi

Cheyletia wells

Cheyletia sp.

Cheyletidae

Clavidromus jackmickleyi

Clavidromus transvaalensis

Cunaxidae

Cydnodromella alveolaris

Czenspinskia sp.

Daidalotarsonemus sp.

Dendrocheyla wellsi

Eriophyidae

Eryngiopus sp.

Euseius alatus

Euseius citrifolius

Euseius concordis

Euseius flechtmanni
Euseius paraguayensis

Eutigmaeus sp.

Eutetranychus banksi

Eutetranychus clarki

Eutetranychus mexicanus

Eutetranychus rusti

Exothorhis caudata

Fungitarsonemus sp.

Galendromimus (Galendromimus) alveolaris

Galendromimus alveolaris

Galendromus (Galendromus) annectens

Galendromus annectens

Galendromus sp.

Hemicheyletia sp.

Hemicheyletia wellsi

Hemitarsonemus latus

Histiostomatidae

Homeopronematus sp.

Iphiseiodes sp.

Iphiseiodes zuluagai

Kampimodromus transvaalensis

Ledermuelleria sp.

Lorryia boyce

Lorryia curiosa

Lorryia formosa

Lorryia spp.

Mediolata sp.

Melissotydeus sp.

Metaseiulus camelliae

Mixonychus sp.

Mononychellus sp.

Neolorryia boycei

Neolorryia sp.

Neoseiulus anonymus

Neoseiulus idaeus

Neoseiulus transvaalensis

Neoseiulus tunus

Neotarsonemus latus

Neotetranycus banksi

Neotropacarus sp.

Oligonychus coffeae

Oligonychus gossypii

Oligonychus merwei

Oribatida

Oribatulidae

Oripodidae

Oulenzia sp.

Paracheyletia wellsi

Parapronematus acaciae

Parapronematus spp.

Paratetranychus bioculatus

Paratetranychus gossypii

Paratetranychus terminalis

Pausia sp.

Phyllocoptruta seringueirae

Phytoscutus sexpilis

Phytoseiidae

Pirnodus sp.

Polyphagotarsonemus latus

Pretydeus curiosa

Pretydeus sp.

Pronematus spp.

Pronematus ubiquitus

Proprioseiopsis dominigos

Proprioseiopsis ovatus

Pseudobonzia sp.

Pseudolorryia cf. nicaraguensis

Pulaeus sp.

Retetydeus boycei

Scutopalus sp.

http://www.biotaneotropica.org.br 
Septanychus braziliensis

Septanychus mexicanus

Shevtchenkella petiolula

Spinoppia sp.

Stigmaeidae

Tarsonemidae

Tarsonemus confusus

Tarsonemus latus

Tarsonemus spp.

Tenuipalpidae

Tenuipalpus heveae

Tenuipalpus phoenicis

Tetrabdella neotropica

Tetranychidae

Tetranychoides californicus

Tetranychus aduncus

Tetranychus banksi

Tetranychus bioculatus

Tetranychus mexicanus

Tetranychus rusti

Tetranychus sp.

Tetranychus telarius

Tetranychus urticae

Triophtydeus sp.

Tydeidae

Tydeus (Afrotydeus) kenyensis

Tydeus (Tydeus) californicus

Tydeus californicus

Tydeus (Tydeus) costensis

Tydeus sp.

Tydeus ubiquitus

Typhlodromalus aff. horatii

Typhlodromalus feresi

Typhlodromina camelliae

Typhlodromips aff. sinensis

Typhlodromips amilus

Typhlodromips cananeiensis

Typhlodromips tunus

Typhlodromus (Amblyseius) ovatus

Typhlodromus (Anthoseius) transvaalensis

Typhlodromus (Typhlodromus) alveolaris

Typhlodromus alveolaris

Typhlodromus annectens

Typhlodromus camelliae

Typhlodromus jackmickleyi

Typhlodromus pectinatus

Typhlodromus sexpilis

Typhlodromus transvaalensis

Typlodromus (Amblyseius) concordis

Tyrophagus putrescentiae

Tyrophagus sp.

Xenotarsonemus sp.

Winterschmidtiidae

Zetzellia aff. yusti

Zetzellia agistzellia

Zetzellia malvinae

Zetzellia mapuchina

Zetzellia quasagistemas

Zetzellia spp.
Host types of mites found in rubber trees in Brazil:

Acacia sp. (Fabaceae)

Acalypha sp. (Euphorbiaceae)

Arachis hypogaea - peanut (Fabaceae)

Bactris setosa (Arecaceae)

Bromeliaceae.

Camellia sp. (Theaceae)

Cassia bicapsularis (Fabaceae)

Cassia sp. (Fabaceae)

Cattleya sp. (Orchidaceae)

Cephaelis sp. (Rubiaceae)

Citrus paradisi - grapefruit (Rutaceae).

Citrus sinensis (Rutaceae)

Citrus sp. - (Rutaceae)

Coffea arabica - coffee (Rubiaceae)

Cydonia sp. - quince (Rosaceae)

Datura sp. (Solanaceae)

Delphinium belladonna (Ranunculaceae)

Desmodium sp. (Fabaceae)

Fleurya aestuans - nettle (Urticaceae)

Gossypium herbaceum - cotton (Malvaceae)

Hevea brasiliensis (Euphorbiaceae)

Ligustrum sp. (Oleaceae)

Mabea fistulifera (Euphorbiaceae)

Mangifera sp. - mango (Anacardiaceae)

Manihot sp. (Euphorbiaceae)

Maytenus nemorosa (Celastraceae)

Mucuna sp.(Fabaceae)

Musa paradisíaca - banana (Musaceae)

Phoenix sp. (Arecaceae)

Psidium guajava - guava (Mirtaceae)

Quercus sp. (Fagaceae)

Rhododendron indicum - (Ericaceae)

Ricinus communis - castor bean (Euphorbiaceae)

Rubus idaeus L. (Rosaceae)

Spathodea sp. (Bignoniaceae)

Theobroma sp. (Sterculiaceae)

Trema floridana (Ulmaceae) 


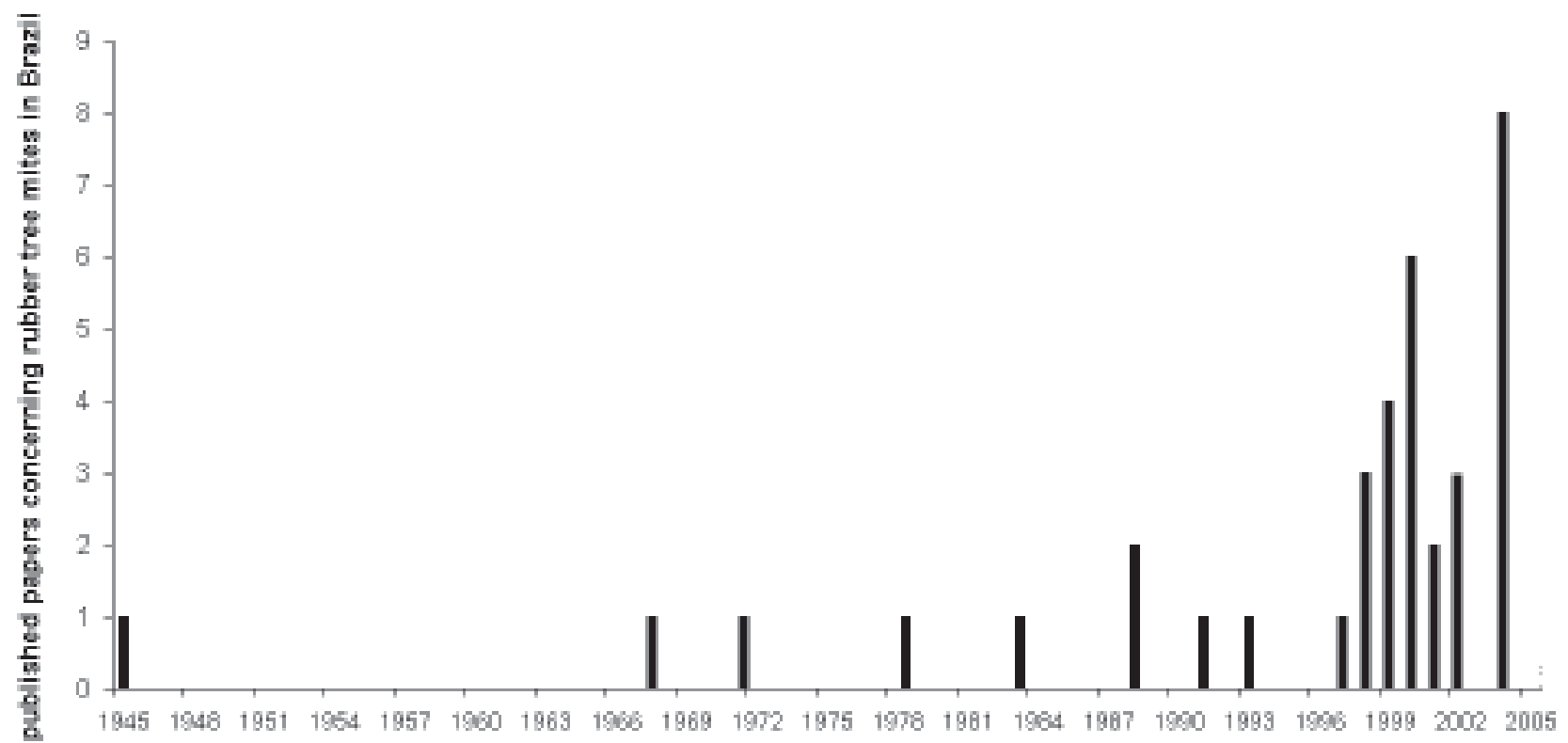

Figure 1. Number of published papers concerning mites on rubber trees in Brazil.

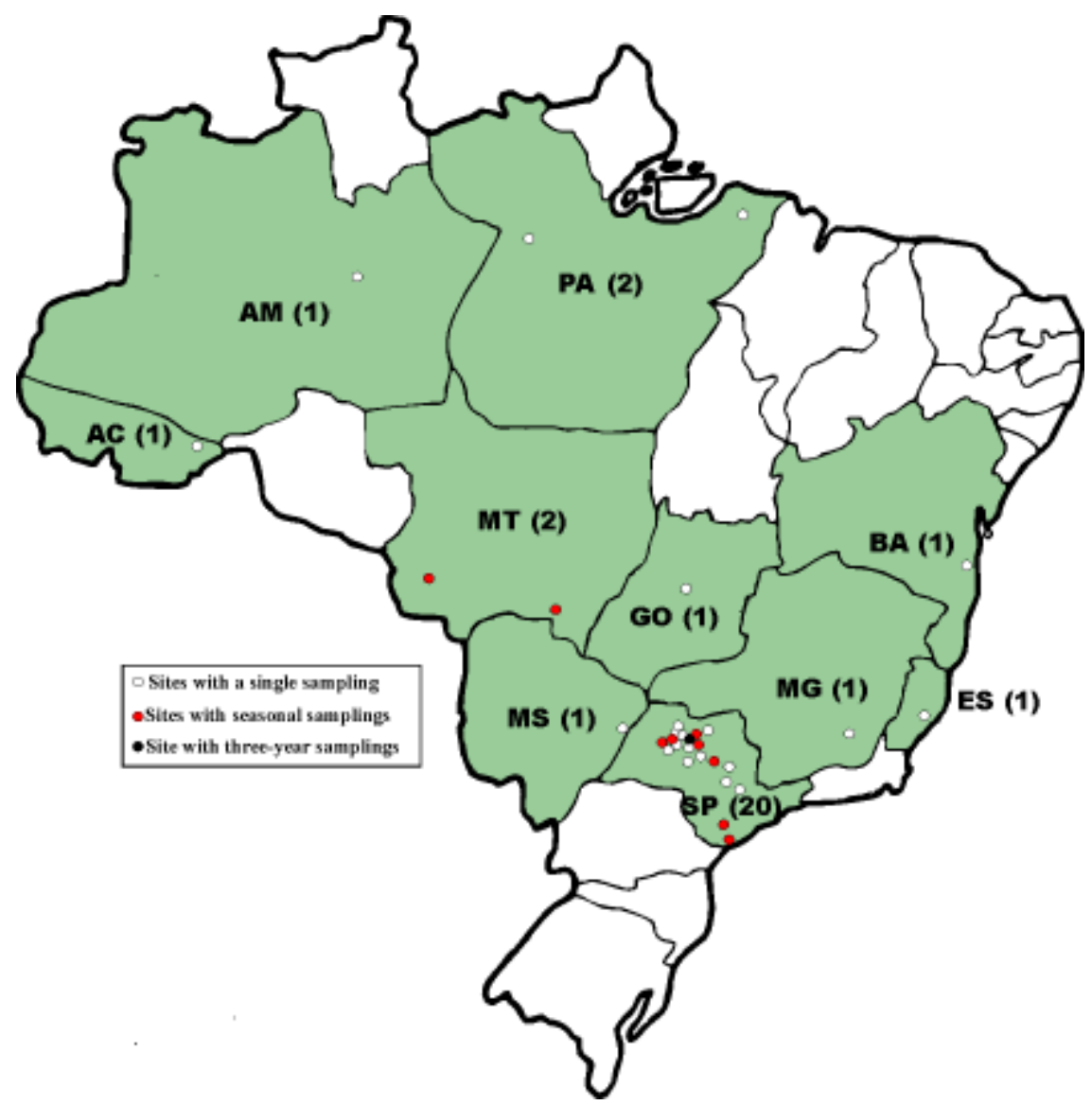

Figure 2. Brazilian states sampled for rubber tree mites (in green); in parenthesis, the number of sites sampled in each state. 